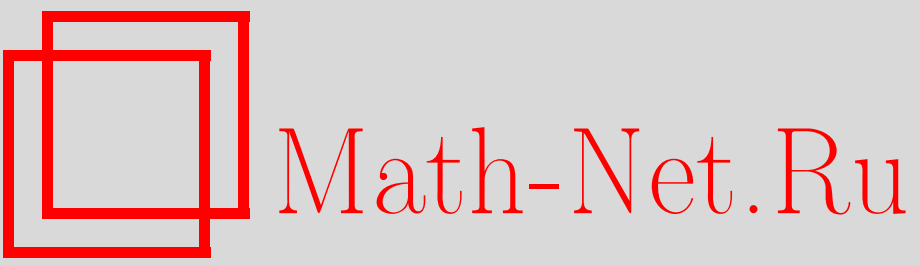

В. И. Кукулин, О. А. Рубщова, Дискретная квантовая теория рассеяния, ТМФ, 2003, том 134, номер 3, 460-486

DOI: https://doi.org/10.4213/tmf165

Использование Общероссийского математического портала Math-Net.Ru подразумевает, что вы прочитали и согласны с пользовательским соглашением

http://www.mathnet.ru/rus/agreement

Параметры загрузки:

IP : 54.162 .27 .143

26 апреля 2023 г., 15:33:05 
ТЕОРЕТИЧЕСКАЯ

И МАТЕМАТИЧЕСКАЯ

ФИЗИКА

Том 134, № 3

март, 2003

(C) 2003 г. $\quad$ В. И. Кукулин* , О.А. Рубцова*

\section{ДИСКРЕТНАЯ КВАНТОВАЯ ТЕОРИЯ РАССЕЯНИЯ}

В работе дается формулировка квантовой теории рассеяния в терминах дискретного $L_{2}$-базиса собственных дифференциалов. В гильбертовом пространстве с использованием проекционных операторов развит универсальный метод построения конечномерных аналогов основных операторов теории рассеяния: $S$ - и $T$-матриц, резольвент и волновых операторов Меллера, а также основных резольвентных тождеств и уравнения Липпмана-Швингера для $T$-матрицы. Развитый общий формализм дискретной теории рассеяния приводит к удивительно простой вычислительной схеме для очень широкого класса операторов взаимодействия.

Ключевые слова: квантовая теория расеяния, волновые пакеты, функция Грина, волновой оператор, $T$-матрица, дискретизация континуума.

\section{1. ВВЕДЕНИЕ}

Трактовка состояний непрерывного спектра в реальных квантовых системах обычно вызывает намного больше трудностей, чем учет дискретного спектра, из-за сложных граничных условий, которым должны удовлетворять функции непрерывного спектра, особенно в задачах нескольких частиц, где имеется несколько континуумов (в разных подсистемах целой системы), часто налагающихся друг на друга. Это сильно сдерживает прогресс многочисленных исследований в разных приложениях обшей теории рассеяния, таких как исследование упругого и неупругого рассеяния составных частиц, в частности, процессов ионизации в области порогов для атомных и молекулярных столкновений. Проблемы возникают также при точном количественном описании многочастичных ядерных реакций в области порогов развала участвующих частищ и вообще при низких энергиях.

В попытке обойти существующие трудности за последние три десятилетия было предложено множество методов, сводящих задачу о непрерывном спектре к задаче дискретного спектра путем разложения функций рассеяния по подходящему $L_{2}$-базису (например, по базису кулоновских функций [1], [2] или функций гармонического осциллятоpa [3]-[7]), или по резонансным полюсам функции Грина с использованием разложения Миттаг-Леффлера [8]. Широкое распространение получил так называемый $J$-матричный подход [1]-[7], в рамках которого коэффициенты разложений по дискретному

*Научно-исследовательский институт ядерной физики им. Д. В. Скобельцына, Московский государственный университет, Москва, Россия. E-mail: kukulin@nucl-th.sinp.msu.ru 
базису, которые являются функциями энергии в соответствуюшей подсистеме, ишутся на основе общих алгебраических соотношений при каждой энергии (при этом используется трехдиагональная форма матрицы оператора кинетической энергии). В последние годы $J$-матричный метод и его многочисленные вариации были с успехом использованы в задачах электронно-атомных столкновений и при исследовании резонансных полюсов $S$-матрицы [7], а также для описания ядерно-ядерных процессов рассеяния [3]-[6] и т.д. Однако все эти реализации $J$-матричного подхода применялись (почти исключительно) к решению одномерных задач (или задач, сводимых к одномерным [6]). При всем удобстве и простоте этого метода его обобщение на реальные задачи многочастичного непрерывного спектра затруднено из-за весьма сложного асимптотического поведения функций континуума нескольких частиц в разных секторах конфигурационного пространства. В итоге процедура сшивания "внутренних" и асимптотических частей волновых функций, присущая всем $J$-матричным подходам и принципиально простая в двухчастичных и сводимых к ним задачах, становится трудно реализуемой в задачах трех и более частиц выше соответствующих порогов. Вследствие последнего обстоятельства до сих пор, несмотря на многочисленные попытки, не появилось обобщения этого метода на описание многочастичного континуума, в котором имеются двух- и многофрагментные каналы рассеяния. $J$-матричный метод также трудно использовать даже в случае двухчастичного рассеяния, если оператор взаимодействия является неэрмитовым (например, комплексный оптический потенциал). Аналогичные проблемы возникают и при применении других методов, основанных на разложениях волновых функций континуума по дискретному базису [9]-[15].

Однако возможен альтернативньй подход к решению задач рассеяния в $L_{2}$-базисе, основанный на "дискретизации” основных операторов теории рассеяния, предложенной нами в работе [16]. Суть этого метода заключается в том, что весь континуум разбивается на энергетические полосы $\left(\mathcal{E}_{i}-\Delta_{i}, \mathcal{E}_{i}\right)$ относительно малой ширины $\Delta_{i}(i=$ $1, \ldots, N)$, и в пределах каждого такого интервала строятся стационарные волновые пакеты $\left|X_{i}\right\rangle$ и $\left|Y_{i}\right\rangle$ соответственно из функций свободного движения или точных волновых функций рассеяния. Эти волновые пакеты (в функциональном анализе называемые собственными дифференциалами (СД) [17]) ограничены и нормируемы в $L_{2}$-пространстве совершенно аналогично функциям дискретного спектра. В работе [16] в рамках шредингеровской (дифференциальной) формулировки квантовой теории рассеяния были получены конечномерные аппроксимации для оператора Грина и внемассовой $T$-матришы. В настоящей работе на основе “дискретизации” основных интегральных уравнений теории рассеяния удалось сделать переход к представлению собственных дифференциалов (ПСД) вполне универсальным. При этом используется простая техника проекционных операторов, на основе которой вместо интегральных операторов теории рассеяния (таких, как операторы Грина, волновые операторы Меллера, $S$ - и $T$-операторы) строятся их конечномерные аналоги, представляемые матрицами конечного ранга. Это позволяет свести задачу от решения сингулярных интегральных уравнений к простой и универсальной матричной алгебре.

В отличие от $J$-матричных методов, при таком подходе не требуется трехдиагональ- 
ная форма оператора кинетической энергии, так как нет сшивки внутренних и асимптотических решений и базис дискретизации соответственно может быть выбран произвольным образом. Метод ПСД, как будет показано ниже, легко “справляется" с нелокальными, зависящими от энергии и неэрмитовыми операторами взаимодействия. А самое главное, такой подход с дискретизацией интегральных операторов непосредственно обобщается на задачу рассеяния нескольких тел, т.е. на дискретизацию многочастичного континуума, поскольку, как хорошо известно, наиболее удобной формой многочастичных уравнений квантовой теории рассеяния являются именно интегральные уравнения (например, уравнения Фаддеева-Якубовского), содержащие все необходимые граничные условия.

С другой стороны, как было показано в нашей работе [16], в рамках предлагаемого подхода весьма удобно строить базисные векторы $\left|Y_{i}\right\rangle$ и $\left|X_{i}\right\rangle$ путем простой диагонализации соответствующей матрицы полного гамильтониана (или оператора кинетической энергии) в подходящем $L_{2}$-базисе, например, в базисе гармонического осциллятора. Таким образом, данный метод соединяет в себе удобства нахож дения собственных (конечномерных) векторов матришы гамильтониана (что присуше всем методам дискретизации континуума) со значительными преимуществами интегрального подхода к теории рассеяния.

Структура настоящей работы такова. В разделе 2 строятся матричные аналоги уравнений Липпмана-Швингера с использованием плосковолновых СД $\left|X_{i}\right\rangle$. Основой построения является конечномерное представление для свободной резольвенты. В этом же разделе строится дискретный аналог основного оператора теории рассеяния - волнового оператора Меллера. Для полноты картины мы приведем также дискретные представления $S$ - и $T$-операторов. Некоторые вопросы, связанные с их построением в случае комплексного потенциала взаимодействия, вынесены в приложение к данной работе.

Раздел 3 содержит несколько численных иллюстраций применения нашего подхода к разным типам взаимодействия, включая неэрмитовы. Здесь показано, что $L_{2}$-базис относительно малой размерности $(N=10-20)$ позволяет с хорошей точностью определить фазовые сдвиги для действительных и комплексных операторов взаимодействия.

В разделе 4 для удобства читателей мы даем развернутое сравнение предлагаемого подхода с другими методами дискретизации континуума, часто используемыми в литературе, в частности с уже упомянутыми выше $J$-матричными методами, а также с методами построения конечномерных аппроксимаций для функции Грина.

В последнем, пятом, разделе приводятся заключительные замечания, в основном касающиеся дальнейших перспектив обсуждаемого здесь подхода.

\section{2. ОПЕРАТОРЫ ТЕОРИИ РАССЕЯНИЯ В ТЕРМИНАХ ПСД}

2.1. Основные положения метода. Предметом нашего рассмотрения (как и в работе [16]) является двухчастичная задача рассеяния с центральными силами (для простоты). Гамильтониан, описываюший движение частишы во внешнем центрально-симметричном поле (или взаимодействие двух частиц), имеет вид

$$
H=H_{0}+V,
$$


где $H_{0}$ - оператор кинетической энергии, а $V$ - потенциал взаимодействия, который считается короткодействуюшим (случай дальнодействующего потенциала типа кулоновского требует отдельного, хотя и сходного, рассмотрения).

В силу сохранения орбитального момента $l$ и его проекции на ось $z$ будем считать их фиксированными.

Итак, пусть $\left\{\Psi_{0}(E, l)\right\}$ - полный набор собственных состояний оператора $H_{0}$ (ортонормированных на $\delta$-функцию от энергии):

$$
\Psi_{0}(E, l ; r)=\sqrt{\frac{k^{3}}{2 E}} \frac{J_{l+1 / 2}(k r)}{\sqrt{k r}}, \quad k=\sqrt{\frac{2 E m}{\hbar^{2}}},
$$

a $\left\{\Psi^{( \pm)}(E, l)\right\}$ и $\{\Psi(E, l)\}$ - полные наборы состояний непрерывного спектра полного гамильтониана $H$, отвечающие соответственно граничным условиям в виде расходящихся $(+)$, сходяшихся $(-)$ и стоячих волн.

Разобьем весь непрерывный спектр энергий $E \in(0, \infty)$ на подынтервалы $\left(\mathcal{E}_{i-1}, \mathcal{E}_{i}\right)$ такие, что

$$
0=\mathcal{E}_{0}<\mathcal{E}_{1}<\cdots<\mathcal{E}_{i}<\cdots<\mathcal{E}_{N}, \quad \Delta_{i}=\mathcal{E}_{i}-\mathcal{E}_{i-1}, \quad \mathcal{E}_{i}^{*}=\frac{1}{2}\left(\mathcal{E}_{i}+\mathcal{E}_{i-1}\right) .
$$

Здесь весь непрерывный спектр ограничивается максимальным значением энергии $\mathcal{E}_{N}$, однако можно считать, что в пределе $N \rightarrow \infty, \mathcal{E}_{N} \rightarrow \infty$. Теперь построим на каждом таком подынтервале С Д функций свободного движения (стационарный волновой пакет)

$$
X_{i}(r)=\frac{1}{\sqrt{\Delta_{i}}} \int_{\mathcal{E}_{i-1}}^{\mathcal{E}_{i}} \Psi_{0}(E, l ; r) d E
$$

а также СД точных функций рассеяния

$$
\begin{aligned}
Y_{i}(r) & =\frac{1}{\sqrt{\Delta_{i}}} \int_{\mathcal{E}_{i-1}}^{\mathcal{E}_{i}} \Psi(E, l ; r) d E, \\
Y_{i}^{( \pm)}(r) & =\frac{1}{\sqrt{\Delta_{i}}} \int_{\mathcal{E}_{i-1}}^{\mathcal{E}_{i}} \Psi^{( \pm)}(E, l ; r) d E .
\end{aligned}
$$

Легко видеть [16], что эти функции имеют такие же свойства, как и функции дискретного спектра

$$
\begin{aligned}
\left\langle X_{i}\left|H_{0}\right| X_{j}\right\rangle & =\mathcal{E}_{i}^{*} \delta_{i, j}, & \left\langle Y_{i}|H| Y_{j}\right\rangle & =\mathcal{E}_{i}^{*} \delta_{i, j} \\
\left\langle X_{i} \mid X_{j}\right\rangle & =\delta_{i, j}, & \left\langle Y_{i} \mid Y_{j}\right\rangle & =\delta_{i, j}
\end{aligned}
$$

Кроме того, для матричных элементов от $n$-й степени гамильтонианов справедливо соотношение

$$
\left\langle X_{i}^{l}\left|H_{0}^{n}\right| X_{j}^{l}\right\rangle=\left\langle Y_{i}^{l}\left|H^{n}\right| Y_{j}^{l}\right\rangle=\left(\mathcal{E}_{i}^{*}\right)^{n}\left[1+O\left(\left(\frac{\Delta_{i}}{\mathcal{E}_{i}^{*}}\right)^{2}\right)\right] \delta_{i, j}, \quad n=2, \ldots
$$


(В случае вещественного потенциала взаимодействия функции $\left|Y_{i}^{( \pm)}\right\rangle$будут обладать теми же свойствами, что и $\left|Y_{i}\right\rangle$. Случай комплексного потенциала рассмотрен в приложении к данной работе.)

В ограниченной области координатного пространства СД отличаются от точных функций рассеяния лиш нормировочным множителем, а далее быстро убывают (см. [16]). Поэтому, как видно из (6), каждый из наборов состояний $\left\{\left|X_{i}\right\rangle\right\}_{i=1}^{N}$ и $\left\{\left|Y_{i}\right\rangle\right\}_{i=1}^{N}$ (а также $\left\{\left|Y_{i}^{( \pm)}\right\rangle\right\}_{i=1}^{N}$ ) образует ортонормированную систему. Однако для построения согласованного формализма целесообразно выбрать из этих наборов один.

В качестве основного базиса ПСД в этой работе будем использовать конечный набор $L_{2}$-состояний $\left\{\left|X_{i}\right\rangle\right\}_{i=1}^{N}$, образованных из функций свободного движения. Это означает, что вместо всех операторов и волновых функций системы будут рассматриваться их проекции на пространство этих векторов. Проектор на пространство СД $\left\{\left|X_{i}\right\rangle\right\}$, очевидно, имеет вид

$$
P=\sum_{i=1}^{N}\left|X_{i}\right\rangle\left\langle X_{i}\right|
$$

В работе [16] было показано, что в ограниченной области конфигурационного пространства $\left(r, r^{\prime}<R_{\max }\right)$ ядро проектора (8) будет совпадать с ядром оператора

$$
\bar{I}=\int_{0}^{\mathcal{E}_{N}} d E\left|\Psi_{0}(E, l)\right\rangle\left\langle\Psi_{0}(E, l)\right|
$$

с точностью до малых второго порядка относительно ширин $\Delta_{i}$. Таким образом, для проектора $Q$, дополняющего $P$ до единичного, будет верно приблизительное равенство

$$
Q \approx \int_{\mathcal{E}_{N}}^{\infty} d E\left|\Psi_{0}(E, l)\right\rangle\left\langle\Psi_{0}(E, l)\right|
$$

Можно условно сказать (не вдаваясь в математические детали), что $P \rightarrow 1, Q \rightarrow 0$ в пределе $N \rightarrow \infty$.

Благодаря тому, что потенциал считается короткодействующим, в $P$-подпространстве его можно заменить потенциалом

$$
V \mapsto V_{P}=P V P=\sum_{i, j=1}^{N} V_{i j}\left|X_{i}\right\rangle\left\langle X_{j}\right|
$$

где

$$
V_{i j}=\left\langle X_{i}|V| X_{j}\right\rangle
$$

Эта замена является основным приближением нашего метода.

Приведем здесь еще одно полезное свойство функций $\left|X_{i}\right\rangle$, которое нам понадобится в дальнейшем:

$$
\left\langle X_{i} \mid \Psi_{0}(E, l)\right\rangle=\frac{1}{\sqrt{\Delta_{i}}} \int_{\mathcal{E}_{i-1}}^{\mathcal{E}_{i}} d E^{\prime}\left\langle\Psi_{0}\left(E^{\prime}, l\right) \mid \Psi_{0}(E, l)\right\rangle= \begin{cases}\frac{1}{\sqrt{\Delta_{i}}}, & E \in\left(\mathcal{E}_{i-1}, \mathcal{E}_{i}\right), \\ 0, & E \notin\left(\mathcal{E}_{i-1}, \mathcal{E}_{i}\right) .\end{cases}
$$

Таким образом, для любого значения энергии на всем подынтервале $E \in\left(\mathcal{E}_{i-1}, \mathcal{E}_{i}\right)$ имеет место соотношение

$$
P\left|\Psi_{0}(E, l)\right\rangle=\frac{\left|X_{i}\right\rangle}{\sqrt{\Delta_{i}}}
$$


2.2. Оператор Грина гамильтониана свободного движения. В рамках предлагаемого подхода вместо точного оператора Грина $\widehat{G}_{0}^{( \pm)}(E)=\left[(E \pm i 0)-H_{0}\right]^{-1}$, отвечающего гамильтониану свободного движения, будет использоваться его $P$-проекция

$$
\widehat{G}_{0 P}^{( \pm)}(E) \equiv P \widehat{G}_{0}^{( \pm)}(E) P=\sum_{i, j=1}^{N}\left|X_{j}\right\rangle\left\langle X_{j}\left|\widehat{G}_{0}^{( \pm)}(E)\right| X_{i}\right\rangle\left\langle X_{i}\right|
$$

Для вычисления матричных элементов, стоящих в правой части последнего равенства, воспользуемся точным спектральным разложением оператора Грина [18]:

$$
\left\langle X_{j}\left|\widehat{G}_{0}^{( \pm)}(E)\right| X_{i}\right\rangle=\int_{0}^{\infty} d E^{\prime} \frac{\left\langle X_{j} \mid \Psi_{0}\left(E^{\prime}, l\right)\right\rangle\left\langle\Psi_{0}\left(E^{\prime}, l\right) \mid X_{i}\right\rangle}{(E \pm i 0)-E^{\prime}} .
$$

Интеграл легко вычислить, используя соотношение (12).

В результате для $P$-проекции оператора Грина гамильтониана свободного движения получаем простое выражение

$$
\widehat{G}_{0 P}^{( \pm)}(E)=\sum_{i=1}^{N} G_{0 i}^{( \pm)}(E)\left|X_{i}\right\rangle\left\langle X_{i}\right|
$$

где комплексные коэффициенты $G_{0 i}^{( \pm)}(E)$ даются точнымми формулами

$$
\begin{aligned}
& \operatorname{Re} G_{0 i}^{( \pm)}(E)=\frac{1}{\Delta_{i}} \ln \left|\frac{\mathcal{E}_{i-1}-E}{\mathcal{E}_{i}-E}\right|, \\
& \operatorname{Im} G_{0 i}^{( \pm)}(E)= \begin{cases}\mp \frac{i \pi}{\Delta_{i}} \delta_{i, j}, & E \in\left(\mathcal{E}_{j-1}, \mathcal{E}_{j}\right), \\
0, & E \geqslant \mathcal{E}_{N} .\end{cases}
\end{aligned}
$$

2.3. Уравнение Липпмана-Швингера для функции рассеяния. Рассмотрим далее точные интегральные уравнения для волновых функций рассеяния, отвечающих граничным условиям в форме расходящихся или сходящихся волн:

$$
\left|\Psi^{( \pm)}(E, l)\right\rangle=\left|\Psi_{0}(E, l)\right\rangle+\widehat{G}_{0}^{( \pm)}(E) V\left|\Psi^{( \pm)}(E, l)\right\rangle .
$$

Используем здесь аппроксимацию потенциала (11) и будем рассматривать эти уравнения при $0<E \leqslant \mathcal{E}_{N}$. Подействовав на (17) слева проектором $P$, получим уравнение для функции $P\left|\Psi^{( \pm)}(E, l)\right\rangle$

$$
P\left|\Psi^{( \pm)}(E, l)\right\rangle=\frac{\left|X_{i}\right\rangle}{\sqrt{\Delta_{i}}}+\widehat{G}_{0 P}^{( \pm)}(E) V_{P} P\left|\Psi^{( \pm)}(E, l)\right\rangle, \quad E \in\left(\mathcal{E}_{i-1}, \mathcal{E}_{i}\right),
$$

которое представляет собой матричный (конечномерный) аналог интегрального уравнения Липпмана-Швингера.

Будем далее считать, что $E \in\left(\mathcal{E}_{i-1}, \mathcal{E}_{i}\right)$. Введем обозначения для $P$-проекций функций рассеяния:

$$
\begin{aligned}
|\mathcal{Y}(E)\rangle & \equiv P|\Psi(E, l)\rangle \sqrt{\Delta_{i}}, \\
\left|\mathcal{Y}^{( \pm)}(E)\right\rangle & \equiv P\left|\Psi^{( \pm)}(E, l)\right\rangle \sqrt{\Delta_{i}},
\end{aligned}
$$

5 Теоретическая и математическая физика, т. 134, № 3, 2003 г. 
или, подробнее,

$$
\left|\mathcal{Y}^{( \pm)}(E)\right\rangle=\sum_{k=1}^{N} \Omega_{k}^{i( \pm)}(E)\left|X_{k}\right\rangle
$$

где

$$
\Omega_{k}^{i( \pm)}(E)=\sqrt{\Delta_{i}}\left\langle X_{k} \mid \Psi^{( \pm)}(E, l)\right\rangle .
$$

Здесь индекс $i$ указывает на принадлежность энергетическому подынтервалу $\left(\mathcal{E}_{i-1}, \mathcal{E}_{i}\right)$. Тогда из (18) получается система уравнений для коэффициентов $\Omega_{k}^{i( \pm)}(E)$ :

$$
\Omega_{k}^{i( \pm)}(E)=\delta_{k i}+\sum_{n=1}^{N} G_{0 k}^{( \pm)}(E) V_{k n} \Omega_{n}^{i( \pm)}(E), \quad k=1, \ldots, N
$$

Легко видеть, что эти коэффициенты образуют $i$-столбец матрицы

$$
\mathbf{\Omega}^{( \pm)}(E)=\left(\mathbf{1}-\mathbf{G}_{0 P}^{( \pm)}(E) \mathbf{V}\right)^{-1}
$$

(т.е. $\Omega_{k}^{i( \pm)}(E)=\Omega_{k i}^{( \pm)}(E)$ при $\left.E \in\left(\mathcal{E}_{i-1}, \mathcal{E}_{i}\right)\right)$. Здесь $\mathbf{V}$ и $\mathbf{G}_{0 P}^{( \pm)}(E)$ - матрицы соответствуюших операторов в ПСД $\left|X_{i}\right\rangle$. Таким образом, легко получить состояния $\left|\mathcal{Y}^{( \pm)}(E)\right\rangle$, используя соотношения (15) для элементов матришы оператора Грина.

Исследуем теперь, какими свойствами обладают построенные проекции точных функций рассеяния.

В первую очередь отметим, что состояния $\left|\mathcal{Y}^{( \pm)}(E)\right\rangle$ не ортогональны друг другу в обшем случае (в силу неполноты проектора $P$ ). Однако, как мы увидим, эти функции содержат всю необходимую информацию о рассеянии.

В случае вешественного потенциала взаимодействия можно воспользоваться известной связью между функциями рассеяния, отвечаюшими граничным условиям различного типа:

$$
\left|\Psi^{( \pm)}(E, l)\right\rangle=e^{ \pm i \delta_{l}(E)}|\Psi(E, l)\rangle,
$$

где $\delta_{l}(E)$ - парциальные фазовые сдвиги. Аналогичные соотношения, очевидно, будут выполняться для $P$-проекций функций рассеяния:

$$
\left|\mathcal{Y}^{( \pm)}(E)\right\rangle=e^{ \pm i \delta_{l}(E)}|\mathcal{Y}(E)\rangle
$$

В силу (23), а также ортонормированности вешественной системы базисных функций $\left\{\left|X_{i}\right\rangle\right\}_{i=1}^{N}$ и определения (21) элементов $\Omega_{k}^{i( \pm)}(E)$, последние можно записать в следующем виде (в случае действительного потенциала взаимодействия):

$$
\Omega_{m}^{i( \pm)}(E)=e^{ \pm i \delta_{l}(E)} \cdot O_{m}^{i}(E), \quad m=1, \ldots, N
$$

где $O_{m}^{i}(E)$ - действительные числа (которые, очевидно, отвечают вешественным функциям $|\mathcal{Y}(E)\rangle)$. 
Таким образом, для нахождения фазовых сдвигов при рассеянии на вещественном потенциале достаточно знать лишь элементы $\Omega_{m}^{i(+)}(E)$ (или $\left.\Omega_{m}^{i(-)}(E)\right)$, причем

$$
\operatorname{tg} \delta_{l}(E)= \pm \frac{\operatorname{Im} \Omega_{1}^{i( \pm)}(E)}{\operatorname{Re} \Omega_{1}^{i( \pm)}(E)}=\cdots= \pm \frac{\operatorname{Im} \Omega_{m}^{i( \pm)}(E)}{\operatorname{Re} \Omega_{m}^{i( \pm)}(E)}=\cdots= \pm \frac{\operatorname{Im} \Omega_{N}^{i( \pm)}(E)}{\operatorname{Re} \Omega_{N}^{i( \pm)}(E)}
$$

Знаки перед дробями в последних равенствах соответствуют верхним индексам элементов матриц $\boldsymbol{\Omega}^{( \pm)}(E)$.

С другой стороны, можно воспользоваться соотношениями (24) для нахождения элементов $S$-матрищы. Согласно [18]

$$
\left\langle\Psi^{(-)}(E, l) \mid \Psi^{(+)}\left(E^{\prime}, l\right)\right\rangle=\delta\left(E^{\prime}-E\right) S_{l}(E)
$$

где $S_{l}(E)=e^{2 i \delta_{l}(E)}$. Легко видеть, что для элемента $S_{l}(E)$ получается простое соотношение

$$
S_{l}(E)=\frac{\left\langle\mathcal{Y}^{(-)}(E) \mid \mathcal{Y}^{(+)}(E)\right\rangle}{\left\langle\mathcal{Y}^{(-)}(E) \mid \mathcal{Y}^{(-)}(E)\right\rangle} .
$$

Используя далее представление (20), получаем

$$
S_{l}(E)=\frac{\sum_{k=1}^{N} \Omega_{k}^{* i(-)}(E) \Omega_{k}^{i(+)}(E)}{\sum_{k=1}^{N} \Omega_{k}^{* i(-)}(E) \Omega_{k}^{i(-)}(E)} .
$$

Легко показать, что эти соотношения эквивалентны (26) в случае вешественного потенциала взаимодействия. В приложении будет показано, что для элементов $S$-матрицы, отвечающей комплексному потенциалу взаимодействия, верны те же соотношения (29) (тогда как (26) уже не будут выполняться).

В заключение мы хотели бы подчеркнуть, что найденные соотношения для $S$-матрицы и фазовых сдвигов являются точными д для усеченного потенциала $V_{P}$. Таким образом, ошибки нахождения наблюдаемых величин для исходного потенциала $V$ связаны только с заменой (11).

2.4. $T$-матрица. В теории рассеяния $T$-оператор обычно находится из уравнения

$$
\widehat{T}(E)=V+\widehat{T}(E) \widehat{G}_{0}^{(+)}(E) V
$$

или из эквивалентного ему соотношения

$$
\widehat{T}=V+V \widehat{G}^{(+)}(E) V
$$

Из уравнения (31) видно, что для короткодействующего потеншиала (11) оператор $\widehat{T}$ также является "короткодействуюшим", и его можно записать в конечномерном виде

$$
\widehat{T}(E) \approx P \widehat{T}(E) P \equiv \widehat{T}_{P}(E) .
$$


Поэтому из уравнения (30) получаем

$$
\widehat{T}_{P}(E)=V_{P}+\widehat{T}_{P}(E) \widehat{G}_{0}^{(+)}(E) V_{P}
$$

Введем обозначение для матрицы $T$-оператора в ПС Д:

$$
T_{k m}(E) \equiv\left\langle X_{k}\left|\widehat{T}_{P}(E)\right| X_{m}\right\rangle .
$$

Переписывая уравнение (33) в матричной форме и решая его с помощью обрашения матриц, для каждого значения энергии $E$ получим

$$
\mathbf{T}(E)=\mathbf{V} \cdot \mathbf{\Omega}^{( \pm)}(E),
$$

где матрицы $\boldsymbol{\Omega}^{( \pm)}(E)$ определяются из $(22)$. Как известно, элементы обобщенной внемассовой $T$-матрицы определяются соотношением

$$
T_{l}\left(E_{1}, E_{2} ; E\right)=\left\langle\Psi_{0}\left(E_{1}, l\right)|\widehat{T}(E)| \Psi_{0}\left(E_{2}, l\right)\right\rangle \approx\left\langle\Psi_{0}\left(E_{1}, l\right)\left|\widehat{T}_{P}(E)\right| \Psi_{0}\left(E_{2}, l\right)\right\rangle .
$$

Согласно свойству (13), матричный элемент оператора $\widehat{T}_{P}$ не будет меняться при изменении энергии $E_{1}$ на произвольном подынтервале $\left(\mathcal{E}_{k-1}, \mathcal{E}_{k}\right)$ и $E_{2}$ на $\left(\mathcal{E}_{m-1}, \mathcal{E}_{m}\right)$, поэтому на этих подынтервалах будем рассматривать приближение (36) в срединных точках. Используя (20), находим

$$
T\left(\mathcal{E}_{k}^{*}, \mathcal{E}_{m}^{*} ; E\right) \approx\left\langle\Psi_{0}\left(\mathcal{E}_{k}^{*}, l\right)\left|\widehat{T}_{P}(E)\right| \Psi_{0}\left(\mathcal{E}_{m}^{*}, l\right)\right\rangle=\frac{T_{k m}(E)}{\sqrt{\Delta_{k} \Delta_{m}}}=\frac{1}{\sqrt{\Delta_{k} \Delta_{m}}} \sum_{n=1}^{N} V_{k n} \Omega_{n m}^{(+)}(E) .
$$

Здесь мы использовали свойство (12). Как видно, для вычисления элементов обобщенной внемассовой $T$-матрицы нужны также "внемассовые" элементы матриц $\boldsymbol{\Omega}^{(+)}(E)$ (т.е. те, которые не совпадают с элементами $\Omega_{k}^{i(+)}$, входящими в разложение $(20)$ ).

Элементы “полувнемассовой” $T$-матришы получаются из определения (36) при $E_{2}=E$ :

$$
T\left(\mathcal{E}_{k}^{*}, \mathcal{E}_{i}^{*} ; \mathcal{E}_{i}^{*}\right) \approx \frac{T_{k i}\left(\mathcal{E}_{i}^{*}\right)}{\sqrt{\Delta_{k} \Delta_{i}}}=\frac{1}{\sqrt{\Delta_{k} \Delta_{i}}} \sum_{n=1}^{N} V_{k n} \Omega_{n i}^{(+)}\left(\mathcal{E}_{i}^{*}\right)=\frac{1}{\sqrt{\Delta_{k} \Delta_{i}}} \sum_{n=1}^{N} V_{k n} \Omega_{n}^{i(+)}\left(\mathcal{E}_{i}^{*}\right) .
$$

В последние соотношения уже входят “физические” элементы матриц $\boldsymbol{\Omega}^{( \pm)}(E)$. Заметим, что для элементов “полувнемассовой” $T$-матрицы имеется определение, эквивалентное (36) (см. [18]):

$$
T\left(\mathcal{E}_{k}^{*}, \mathcal{E}_{i}^{*} ; \mathcal{E}_{i}^{*}\right)=\left\langle\Psi_{0}\left(\mathcal{E}_{k}^{*}, l\right)|V| \Psi^{(+)}\left(\mathcal{E}_{i}^{*}, l\right)\right\rangle .
$$

Заменяя в соотношении (39) $V$ на $V_{P}$, используя (20) и упрошая, снова получим соотношения (38).

Наконец, элемент $T$-матрицы на массовой поверхности получается из (36) или (39) при $\mathcal{E}_{k}^{*}=\mathcal{E}_{i}^{*}$ :

$$
T\left(\mathcal{E}_{i}^{*}, \mathcal{E}_{i}^{*} ; \mathcal{E}_{i}^{*}\right) \approx \frac{T_{i i}^{i}}{\Delta_{i}}=\frac{1}{\Delta_{i}} \sum_{n=1}^{N} V_{i n} \Omega_{n}^{i(+)}\left(\mathcal{E}_{i}^{*}\right) .
$$

Таким образом, $T$-матрица как на массовой поверхности, так и вне ее легко находится путем простой свертки матриш $\mathbf{V}$ и $\boldsymbol{\Omega}^{(+)}(E)$. 
2.5. Резольвента полного гамильтониана. Для нахождения $P$-проекции полной резольвенты $\widehat{G}^{( \pm)}(E)$ гамильтониана $H$ в ПСД можно воспользоваться основным резольвентным тождеством

$$
\widehat{G}^{( \pm)}(E)=\widehat{G}_{0}^{( \pm)}(E)+\widehat{G}_{0}^{( \pm)}(E) V \widehat{G}^{( \pm)}(E) .
$$

В случае короткодействуюшего потенциала (11) это уравнение сводится к матричному. Действуя на (41) проектором $P$ справа и слева, получим уравнение для оператора Грина в ПСД $\widehat{G}_{P}^{( \pm)}(E) \equiv P \widehat{G}_{P}^{( \pm)}(E) P$ :

$$
\widehat{G}_{P}^{( \pm)}(E)=\widehat{G}_{0 P}^{( \pm)}(E)+\widehat{G}_{0 P}^{( \pm)}(E) V_{P} \widehat{G}_{P}^{( \pm)}(E)
$$

которое теперь является матричным, а не интегральным. Очень важно, что сингулярность по энергии в этом уравнении при переходе в ПС Д выделяется в явном виде и затем интегрируется, давая несингулярные (логарифмические) коэффициенты (16) (несложно показать, что на концах $i$-го интервала, т.е. при $E=\mathcal{E}_{i}$, логарифмические особенности в $\operatorname{Re} G_{0 i}^{( \pm)}$также являются устранимыми).

Матрица полной резольвенты $\mathbf{G}_{P}^{( \pm)}(E)$ в ПС Д, таким образом, легко находится путем простого матричного умножения:

$$
\mathbf{G}_{P}^{( \pm)}(E)=\mathbf{\Omega}^{( \pm)}(E) \cdot \mathbf{G}_{0 P}^{( \pm)}(E)
$$

2.6. Матричная форма оператора Меллера. В теории рассеяния сушественную роль играют волновые операторы Меллера $\widehat{\Omega}^{( \pm)}$, связывающие точные функции рассеяния и функции свободного движения при заданной энергии:

$$
\left|\Psi^{( \pm)}(E, l)\right\rangle=\widehat{\Omega}^{( \pm)}\left|\Psi_{0}(E, l)\right\rangle .
$$

Эти операторы не зависят от энергии и могут быть записаны в виде спектральной суммы [18] (при фиксированном $l$ ):

$$
\widehat{\Omega}^{( \pm)}=\int_{0}^{\infty} d E\left|\Psi^{( \pm)}(E, l)\right\rangle\left\langle\Psi_{0}(E, l)\right| .
$$

Легко показать, что в ПСД матричные аналоги волновых операторов имеют вид конечных сумм

$$
\widehat{\Omega}_{P}^{( \pm)} \equiv P \widehat{\Omega}^{( \pm)} P=\sum_{i=1}^{N} P\left|Y_{i}^{( \pm)}\right\rangle\left\langle X_{i}\right|,
$$

где $\left|Y_{i}^{( \pm)}\right\rangle$- СД точных функций рассеяния. Используя их определения (5) и (20), сразу получаем

$$
P\left|Y_{i}^{( \pm)}\right\rangle=\frac{1}{\Delta_{i}} \int_{\mathcal{E}_{i-1}}^{\mathcal{E}_{i}}\left|\mathcal{Y}^{( \pm)}(E)\right\rangle d E, \quad i=1, \ldots, N
$$


Теперь становится ясно, что матричные элементы операторов Меллера в ПСД выражаются через элементы $\Omega_{k}^{i( \pm)}(E)$ следующим образом:

$$
\Omega_{m i}^{( \pm)} \equiv\left\langle X_{m}\left|\widehat{\Omega}^{( \pm)}\right| X_{i}\right\rangle=\frac{1}{\Delta_{i}} \int_{\mathcal{E}_{i-1}}^{\mathcal{E}_{i}} \Omega_{m}^{i( \pm)}(E) d E .
$$

При достаточно малых ширинах интервалов $\Delta_{i}$ будут верны приближенные равенства

$$
P\left|Y_{i}^{( \pm)}\right\rangle \approx\left|\mathcal{Y}^{( \pm)}\left(\mathcal{E}_{i}^{*}\right)\right\rangle, \quad \Omega_{m i}^{( \pm)} \approx \Omega_{m}^{i( \pm)}\left(\mathcal{E}_{i}^{*}\right)=\Omega_{m i}^{( \pm)}\left(\mathcal{E}_{i}^{*}\right)
$$

где элементы $\Omega_{m i}^{( \pm)}\left(\mathcal{E}_{i}^{*}\right)$ определяются согласно (22). Таким образом, становится понятным смысл элементов этих матриц $\boldsymbol{\Omega}^{( \pm)}(E)$, входящих в разложение $(20)$ для векторов $\left|\mathcal{Y}^{( \pm)}(E)\right\rangle$ : в средних точках энергетических подынтервалов они соответствуют элементам матриц операторов Меллера. Полные матрицы волновых операторов в ПСД имеют вид

$$
\boldsymbol{\Omega}^{( \pm)}=\left(\begin{array}{ccccc}
\Omega_{11}^{( \pm)}\left(\mathcal{E}_{1}^{*}\right) & \ldots & \Omega_{1 i}^{( \pm)}\left(\mathcal{E}_{i}^{*}\right) & \ldots & \Omega_{1 N}^{( \pm)}\left(\mathcal{E}_{N}^{*}\right) \\
\ldots \ldots \ldots & \ldots & \ldots \ldots \ldots \ldots & \ldots & \ldots \ldots \ldots \\
\Omega_{m 1}^{( \pm)}\left(\mathcal{E}_{1}^{*}\right) & \ldots & \Omega_{m i}^{( \pm)}\left(\mathcal{E}_{i}^{*}\right) & \ldots & \Omega_{m N}^{( \pm)}\left(\mathcal{E}_{N}^{*}\right) \\
\ldots \ldots \ldots & \ldots & \ldots \ldots \ldots \ldots \ldots \ldots \ldots \ldots \ldots \\
\Omega_{N 1}^{( \pm)}\left(\mathcal{E}_{1}^{*}\right) & \ldots & \Omega_{N i}^{( \pm)}\left(\mathcal{E}_{i}^{*}\right) & \ldots & \Omega_{N N}^{( \pm)}\left(\mathcal{E}_{N}^{*}\right)
\end{array}\right)
$$

Таким образом, из набора СД функций свободного движения $\left\{\left|X_{i}\right\rangle\right\}$ можно построить наборы $P$-проекций С Д точных функций рассеяния $\left\{P\left|Y_{i}^{( \pm)}\right\rangle\right\}$, используя матрицы волновых операторов Меллера $\boldsymbol{\Omega}^{( \pm)}$.

Для полноты картины приведем здесь конечномерное представление для $S$-оператора, определяемого соотношением

$$
\widehat{S}=\widehat{\Omega}^{(-) \dagger} \widehat{\Omega}^{(+)}
$$

Матрица $S$ связана с $\widehat{S}$ следуюшим образом:

$$
\left\langle\Psi_{0}(E, l)|\widehat{S}| \Psi_{0}\left(E^{\prime}, l\right)\right\rangle=\left\langle\Psi^{(-)}(E, l) \mid \Psi^{(+)}\left(E^{\prime}, l\right)\right\rangle=\delta\left(E^{\prime}-E\right) S_{l}(E) .
$$

Несложно показать, что в $P$-пространстве оператору $\widehat{S}$ будет отвечать конечная сумма

$$
\widehat{S}_{P} \equiv P \widehat{S} P=\sum_{i=1}^{N}\left|X_{i}\right\rangle S_{i}\left\langle X_{i}\right|,
$$

где

$$
S_{i}=\left\langle Y_{i}^{(-)} \mid Y_{i}^{(+)}\right\rangle
$$

Используя приближенные равенства (49), а также свойства состояний $\left|\mathcal{Y}^{( \pm)}(E)\right\rangle$, можно получить следуюшие приближенные соотношения для дискретного аналога оператоpa $\widehat{S}$ :

$$
\widehat{S}_{P}=\sum_{i=1}^{N}\left|X_{i}\right\rangle \frac{\left\langle\mathcal{Y}^{(-)}\left(\mathcal{E}_{i}^{*}\right) \mid \mathcal{Y}^{(+)}\left(\mathcal{E}_{i}^{*}\right)\right\rangle}{\left\langle\mathcal{Y}^{(-)}\left(\mathcal{E}_{i}^{*}\right) \mid \mathcal{Y}^{(-)}\left(\mathcal{E}_{i}^{*}\right)\right\rangle}\left\langle X_{i}\right| .
$$


В заключение этого раздела будет полезно кратко резюмировать полученные результаты. Формализм СД был ранее использован ([17], [19]) для строгого доказательства основных свойств операторов и собственных состояний (таких, как эрмитовость, полнота и т.д.), относящихся к непрерывному спектру энергий в квантовых системах, с целью формулировки задачи рассеяния в рамках теории операторов в гильбертовом пространстве. В настоящей работе мы обобщили этот формализм и сушественно расширили сферу его применения на основные уравнения квантовой теории рассеяния.

Кроме того, был сфформулирован общий алгоритм “дискретизации” операторов теории рассеяния, таких как $S$ - и $T$-матрицы, волновой оператор Меллера и резольвента. Одним из основных преимушеств найденных дискретных представлений является простота и универсальность всех вычислений в ПСД. В частности, если использовать $L_{2}$-базис для разложения векторов $\left|X_{i}\right\rangle$ и $\left|Y_{i}\right\rangle$, то, как показано в [16], всего одна диагонализация матрицы полного гамильтониана в таком базисе позволяет найти наблюдаемые величины для широкого энергетического интервала.

Следующий раздел будет посвящен проверке сходимости наблюдаемых величин, найденных с помошью “дискретизованных” операторов конечного ранга.

\section{3. ПРИМЕРЫ}

3.1. Построение СД точных функций рассеяния. Для проверки сходимости метода построения СД $|\mathcal{Y}(E)\rangle$ с помошью матриц оператора Меллера $\Omega^{( \pm)}(E)$ мы использовали две различные реализации $L_{2}$-базиса состояний $\left|X_{i}\right\rangle$.

A. Конечный базис функций гармонического осциллятора. В нашей предыдушей работе [16] было показано, что "на конечном носителе" можно отождествить СД функций свободного движения $\left|X_{i}\right\rangle$ с квадратично-интегрируемыми функциями, полученными при диагонализации матрищы свободного гамильтониана $H_{0}$ в дискретном базисе, если набор срединных точек разбиения $(3)\left\{\mathcal{E}_{i}^{*}\right\}$ будет совпадать с полученным набором собственных значений. В качестве такого базиса мы использовали конечный набор волновых функций гармонического осциллятора. Таким образом, в ограниченной области пространства можно записать

$$
X_{i}(r) \approx \sum_{n=0}^{N-1} C_{i n} R_{n l}(r), \quad i=1, N,
$$

где $R_{n l}=|n l\rangle$ - радиальная часть функции гармонического осциллятора. Коэффициенты $\left\{C_{i n}^{l}\right\}$ и значения $\left\{\mathcal{E}_{i}^{*}\right\}_{i=1}^{N}$ представляют собой собственные векторы и собственные значения матрицы свободного гамильтонинана $\left\|\left\langle n l\left|H_{0}\right| n^{\prime} l\right\rangle\right\|$. Заметим, что в этом случае для оператора проектирования будет верно представление

$$
P=\sum_{n=0}^{N-1}|n l\rangle\langle n l| .
$$

Используя (54) и (55), полученные ранее выражения для функций, операторов рассеяния и уравнений в ПСД легко переписать в осцилляторном представлении (в подпространстве $n \leqslant N-1$ ). 
Рассмотрим для иллюстрации простой случай, когда потенциал взаимодействия является локальным и имеет вид

$$
V(r)=-V_{0} e^{-\gamma r^{2}}
$$

при этом безразмерный параметр силы потенциала можно определить как

$$
\xi=\frac{\hbar^{2} \gamma}{m V_{0}}
$$

Построение узловых точек $\mathcal{E}_{i}$ и ширин $\Delta_{i}$ при разбиении спектра (3), а также вычисление элементов $V_{i k}$ (имеющих аналитический вид) подробно описаны в работе [16]. Таким образом, вычисление элементов матриц $\boldsymbol{\Omega}^{( \pm)}(E)(22)$ не составляет особого труда.

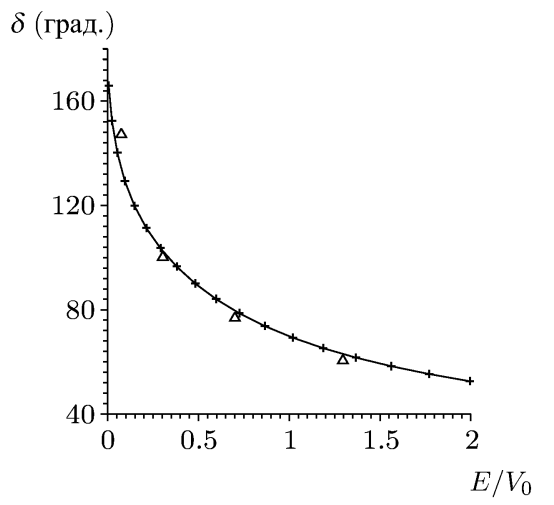

a

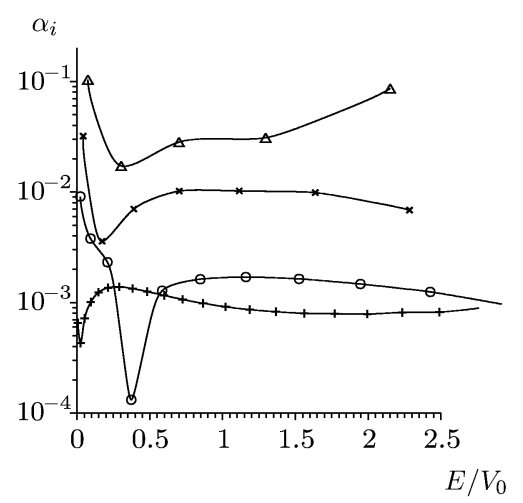

б

Рис. 1

При вычислениях с осцилляторными функциями удобно использовать безразмерный параметр $q=r_{\omega} \sqrt{\gamma}$, где $r_{\omega}=\sqrt{\hbar /(m \omega)}-$ осцилляторный радиус. Параметр $r_{\omega}$ является произвольным радиальным масштабом выбранного базиса, и его естественно выбрать из соображений наилучшей сходимости вычисляемых величин при фиксированном количестве базисных функций $N$.

Мы построили матрицы $\boldsymbol{\Omega}^{( \pm)}(E)$ и проверили соотношения (26) для срединных точек энергетических интервалов $\left(\mathcal{E}_{i-1}, \mathcal{E}_{i}\right)$ в пределах по энергии $0-2 V_{0}$. Расчеты проводились для параметров потенциала $V_{0}=41.47 \mathrm{MэB}, \gamma=0.2 \Phi_{\mathrm{M}}^{-2}, \xi=0.2$. Точность выполнения этих соотношений оказалась $\sim 10^{-15}$ (что практически совпадает с точностью компьютерных вычислений) для любой размерности базиса. Кроме того, $S$ и $T$-матрицы, найденные с помощью матриц $\boldsymbol{\Omega}^{( \pm)}(E)$, строго удовлетворяют условиям унитарности (в отличие от аппроксимаций, предложенных нами в работе [16] в рамках шредингеровского формализма). На рис. $1 a$ представлены графики зависимости от энергии фаз рассеяния $\delta_{l}\left(\mathcal{E}_{i}^{*}\right)$, найденных из соотношений $(26)$, для разных значений размерности базиса $(N=6(\triangle), N=41(+))$ при $l=0$. Для сравнения на графики 
наложены "точные" значения фаз (сплошная линия), полученные с помощью процедуры численного интегрирования уравнения Шредингера для потенциала (56). На рис. 16 представлены погрешности расчетных фаз относительно “точных" значений

$$
\alpha_{i}=\frac{\delta_{\text {ocц }}\left(\mathcal{E}_{i}^{*}\right)-\delta\left(\mathcal{E}_{i}^{*}\right)}{\delta\left(\mathcal{E}_{i}^{*}\right)}
$$

(при размерностях базиса $N=6(\triangle), N=11(\times), N=21(\circ), N=41(+))$. Хорошо видно, что даже при использовании базиса очень малого размера $(N=6)$ средние ошибки в определении фазовых сдвигов составляют всего несколько процентов. В целом же можно сделать вывод, что уменьшение погрешности на порядок происходит при увеличении размерности базиса примерно в четыре раза.

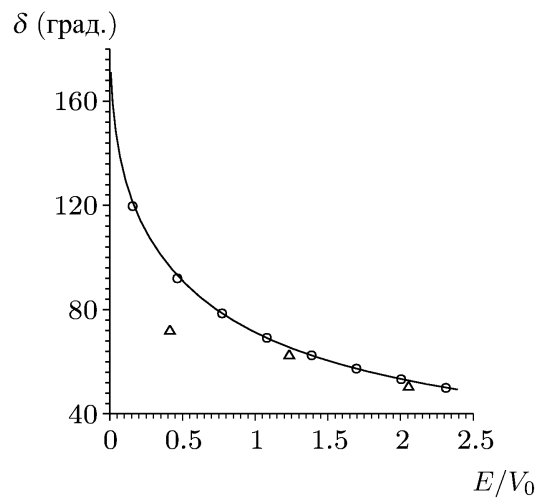

a

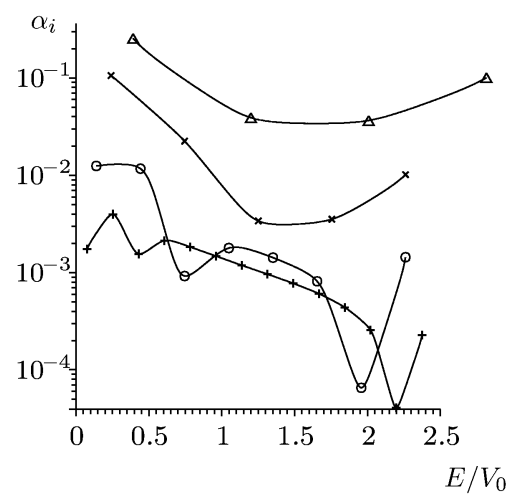

б

Рис. 2

Б. Эквидистантное разбиение спектра. Использование в качестве базисных элементов С Д функций свободного движения позволяет вовсе избежать диагонализации матрицы гамильтониана в каком-либо дискретном базисе.

Рассмотрим эквидистантное разбиение непрерывного спектра

$$
0=\mathcal{E}_{0}<\mathcal{E}_{1}<\cdots<\mathcal{E}_{N}, \quad \Delta=\frac{\mathcal{E}_{N}}{N}, \quad \mathcal{E}_{k}=k \cdot \Delta .
$$

На каждом подынтервале С Д функции свободного движения будут иметь вид

$$
X_{i}(r)=\frac{1}{\sqrt{\Delta}} \int_{\mathcal{E}_{i-1}}^{\mathcal{E}_{i}} d E \sqrt{\frac{k^{3}}{2 E}} \frac{J_{l+1 / 2}(k r)}{\sqrt{k r}}, \quad k=\sqrt{\frac{2 m E}{\hbar^{2}}},
$$

где $J_{l+1 / 2}$ - цилиндрические функции Бесселя.

При $l=0$ можно приближенно записать (в единицах $\hbar=m=1$ )

$$
X_{i}(r) \approx \sqrt{\frac{2 \Delta}{\pi k_{i}^{*}}} \sin \left(k_{i}^{*} r\right) \frac{\sin \left(\frac{\delta k_{i}}{2} r\right)}{\frac{\delta k_{i}}{2} r}, \quad \delta k_{i}=\frac{\Delta}{\sqrt{2 \mathcal{E}_{i}^{*}}},
$$




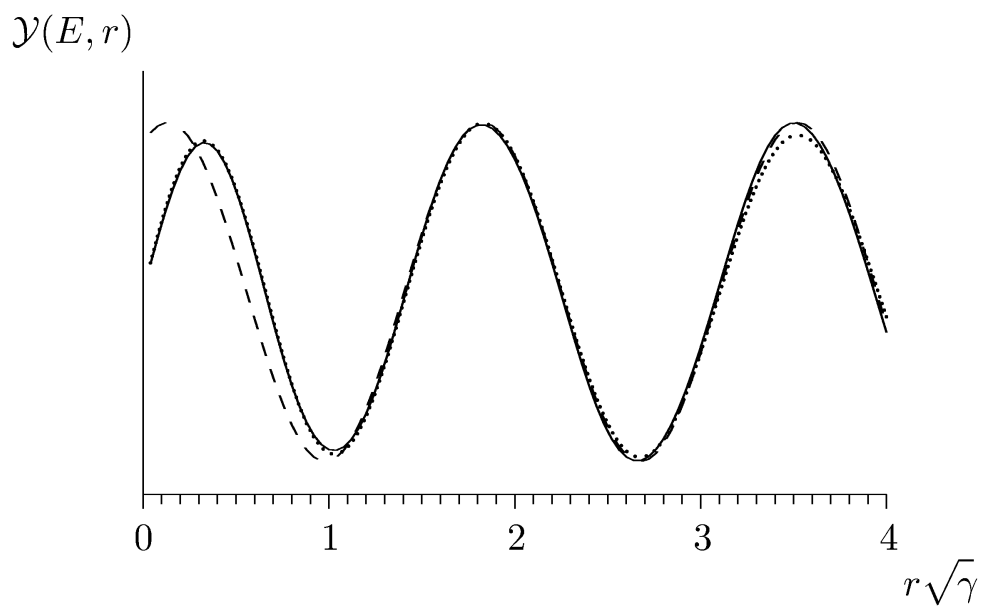

Рис. 3

откуда хорошо видно, что в области $r \ll\left(\delta k_{i}\right)^{-1}$ базисные функции $X_{i}(r)$ ведут себя как плоские волны, однако, взятые во всем пространстве, удовлетворяют условиям нормировки на единицу и взаимной ортогональности.

Элементы матрицы потенциала взаимодействия в ПС Д находятся из приближенного равенства

$$
V_{i k} \approx \frac{\left\langle\Psi_{0}\left(\mathcal{E}_{i}^{*}, l\right)|V| \Psi_{0}\left(\mathcal{E}_{k}^{*}, l\right)\right\rangle}{\Delta} .
$$

Эти матричные элементы имеют аналитический вид для многих типов потенциалов, традиционно используемых в теории рассеяния, в частности для гауссовых и родственых им потенциалов (см. [15], [16]). Элементы $G_{0 k}^{(+)}(E)$ зависят только от узлов и ширин разбиения. Таким образом, матрицы $\boldsymbol{\Omega}^{(+)}(E)(22)$ легко находятся и в этом случае. В таком базисе нами были проверены соотношения (26) для эквидистантного разбиения спектра свободного гамильтониана $H_{0}$. Точность выполнения этих равенств получилась такая же, как и при расчетах в осцилляторном базисе. На рис. $2 a$ представлены зависимости от энергии вычисленных при $l=0$ фаз рассеяния (число учитываемых подынтервалов $N=5(\triangle), N=20($ ( )) в сравнении с "точными" значениями (сплошная линия).

На рис. 26 представлены относительные погрешности найденных фазовых сдвигов

$$
\alpha_{i}=\frac{\delta_{\text {экв }}\left(\mathcal{E}_{i}^{*}\right)-\delta\left(\mathcal{E}_{i}^{*}\right)}{\delta\left(\mathcal{E}_{i}^{*}\right)}
$$

при размерностях базиса $N=5(\triangle), N=10(\times), \quad N=20(\circ)$ и $N=40(+)$. Хорошо видно, что уже при скромных размерах базиса точность нахождения наблюдаемых величин оказывается весьма высокой.

При эквидистантном разбиении спектра основным параметром является максимальное значение энергии $\mathcal{E}_{N}$. Эта величина выбиралась так, чтобы наилучшим образом приблизить фазовые сдвиги при фиксированном числе учитываемых энергетических интервалов $N$. 
На рис. 3 представлены координатные зависимости волновых пакетов $\mathcal{Y}(E, r)$, построенных из базисных функций $X_{i}(r)$ с помошью матриц $\boldsymbol{\Omega}^{( \pm)}(E)$ как с использованием осцилляторного базиса (сплошная линия), так и с помошью базиса (60) (пунктирная линия) при одной и той же энергии $(E=56 \mathrm{MэB})$ в сравнении с их асимптотическим поведением (штриховая линия). Хорошо видно, что для аппроксимации СД волновой функции рассеяния с одинаковым успехом подходят оба типа базисных функций.

3.2. Нахождение наблюдаемых величин в случае комплексного (оптического) потенциала взаимодействия. Сушественными преимушествами представленного в этой работе метода дискретизации континуума при нахождении элементов $T$-матрицы (на основе матричного уравнения Липпмана-Швингера) перед большинством известных нам на настоящий момент методов решения задачи рассеяния в $L_{2}$-базисе [1], [3]-[7], [9]-[15] являются универсальность и гибкость данного подхода. Как следует из вышеизложенного, в качестве базиса дискретизации можно выбрать любой полный набор квадратично-интегрируемых функций, а также много разных типов разбиения непрерывного спектра на отдельные энергетические подынтервалы. Кроме того, не возникает никаких новых проблем при включении мнимых частей потенциалов взаимодействия, расширении класса рассматриваемых взаимодействий на нелокальные и зависящие от энергии и т.д. Причем сложность вычислений в рамках нашей схемы для комплексных и/или нелокальных взаимодействий та же, что и в случае простого действительного потенциала, тогда как $J$-матричные методы в случае оптического потенциала едва ли применимы. Дело в том, что почти все методы дискретизации континуума опираются на диагонализацию конечной матрицы гамильтониана $H$ в выбранном дискретном базисе. В случае комплексного потенциала оператор $H$ уже не является эрмитовым, и подобная диагонализация становится невозможной. Напротив, в “интегральном" подходе, описанном в настояшей работе, используется лиш конечная матрица эрмитова свободного гамильтониана $H_{0}$.

Для иллюстрации этих утверждений мы рассчитали элементы $T$-матрицы на массовой поверхности для комплексного потенциала вида

$$
V(r)=-V_{0} e^{-\gamma r^{2}}-i W_{0} e^{-\eta r^{2}}
$$

Расчеты проводились в базисе вещественных функций гармонического осциллятора. Нахождение матриц $\Omega^{( \pm)}(E)$ подробно описано в предыдушем пункте. Параметры потенциала выбирались таким образом $\left(V_{0}=41.47 \mathrm{MэB}, W_{0}=25 \mathrm{MэB}, \gamma=\eta=0.2 \Phi_{\mathrm{M}}{ }^{-2}\right)$, что теория возмушений для мнимой части потенциала не применима. Используя найденные элементы $T$-матрицы, мы вычислили фазовые сдвиги для $l=0$.

На рис. $4 a$ представлены энергетические зависимости реальных частей фаз для нескольких размерностей базиса $(N=6(\triangle), N=21(\circ))$ в сравнении с "точными" значениями (сплошная линия), полученными, как и ранее, путем численного интегрирования уравнения Шредингера. На рис. 46 представлены мнимые части фазовых сдвигов $(N=6(\triangle), N=11(\times), N=21(\circ))$. Относительные погрешности определения реальных

$$
\alpha(\operatorname{Re} \delta)=\frac{\operatorname{Re} \delta_{\text {ocц }}-\operatorname{Re} \delta}{\operatorname{Re} \delta}
$$




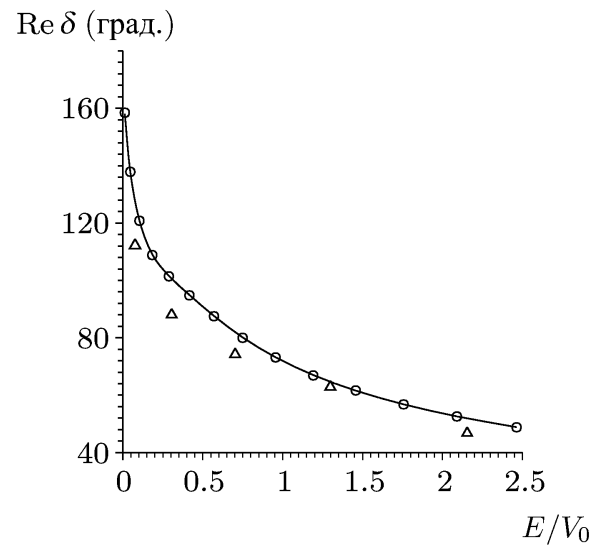

a

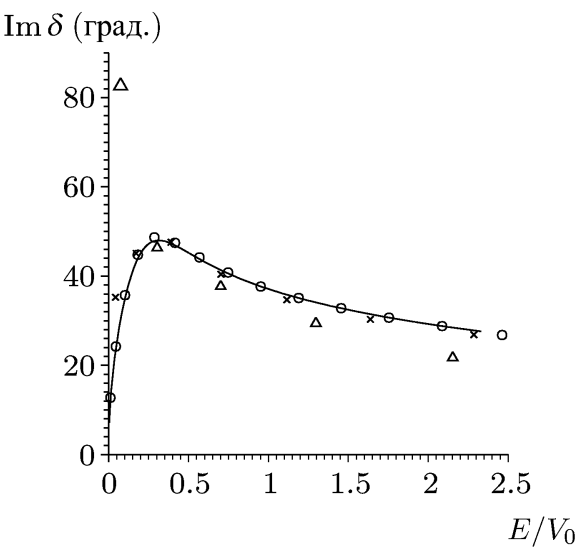

б

Рис. 4

и мнимых

$$
\alpha(\operatorname{Im} \delta)=\frac{\operatorname{Im} \delta_{\text {осц }}-\operatorname{Im} \delta}{\operatorname{Im} \delta}
$$

частей фаз рассеяния представлены на рис. $5 a$ и 56 , соответственно (для размерностей базиса $N=6(\triangle), N=11(\times), N=21(\circ), N=41(+))$. Хорошо видно, что точность вычислений реальных частей фазовых сдвигов примерно та же, что и в случае вешественного потенциала взаимодействия. Погрешности мнимых частей при $N=6$ вблизи порогового значения энергии довольно велики, однако в среднем они составляют несколько процентов.

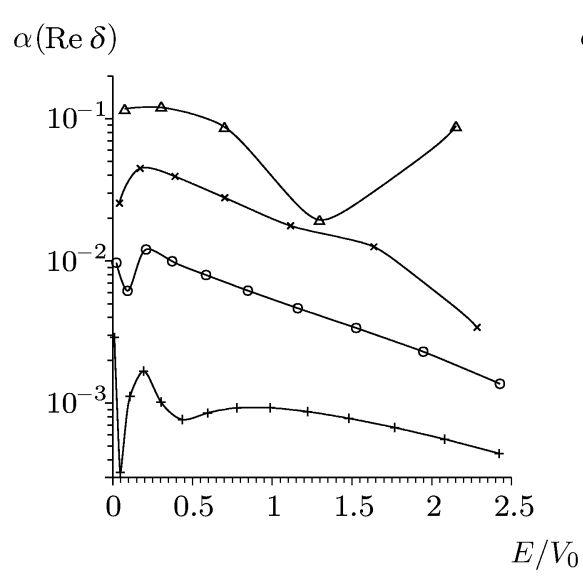

a

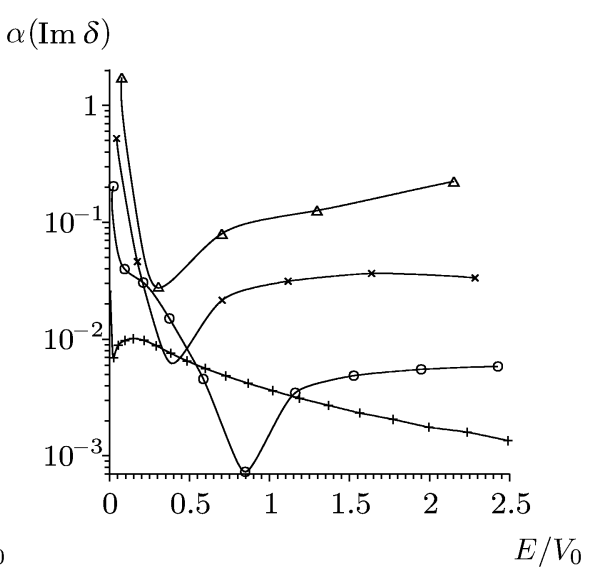

б

Рис. 5 


\section{4. СВЯЗЬ С ДРУГИМИ МЕТОДАМИ ДИСКРЕТИЗАЦИИ КОНТИНУУМА}

$\mathrm{K}$ настоящему времени в разных областях квантовой теории было предложено множество всевозможных методов построения конечномерных представлений как для волновых функций рассеяния, так и для основных операторов теории рассеяния. Поэтому авторы сочли полезным для удобства читателей специально обсудить в данном разделе взаимоотношения между разными методами дискретизации континуума в квантовой теории, чтобы яснее увидеть их сходства и различия. Не в последнюю очередь это намерение мотивировано желанием показать, что даже в тех случаях, когда мы используем для дискретизации тот же осцилляторный базис, что и в других методах, и соответствуюшую процедуру диагонализации матрицы гамильтониана в этом базисе, наш подход к нахождению операторов сильно отличается, например, от так называемого представления гармонического осциллятора (ПГО) [3]-[6] в теории рассеяния. Поэтому ниже мы кратко обсудим основные предлагаемые на сегодняшний момент методы.

4.1. J-матричные методы. K одному из наиболее известных направлений подобных исследований относятся методы $J$-матрицы [1]-[7] (матрицы Якоби). Этот подход, хотя и предложенный около 30 лет назад, приобрел в последние годы большую популярность. Обсудим его на примере ПГО в теории рассеяния. В качестве базиса для разложения функций рассеяния используется полный бесконечный набор собственных функций гармонического осциллятора (см., например, [5]), а качестве операторов теории рассеяния используются их матричные элементы в этом базисе. Основным удобством выбранной системы базисных функций является тот факт, что матриша гамильтониана свободного движения $H_{0}$ трехдиагональна в этом представлении, т.е. представляет собой матрицу Якоби (другие методы $J$-матричного подхода используют представление биортогонального базиса кулоновских функций [2], в котором трехдиагональна матрица гамильтониана $H_{C}$, включающего двухчастичное кулоновское взаимодействие). В силу этого обстоятельства из матричной алгебры и рекуррентных соотношений легко найти (см. [1], [4]) коэффициенты разложения по элементам выбранного базиса для волновых функций свободного движения (или функций рассеяния на кулоновском потеншиале), отвечаюших граничным условиям в виде расходяшихся и сходяшихся волн:

$$
\Psi_{0}^{(+)}(E, l ; r)=\sum_{n=0}^{\infty} S_{n l}(E) R_{n l}(r), \quad \Psi_{0}^{(-)}(E, l ; r)=\sum_{n=0}^{\infty} C_{n l}(E) R_{n l}(r) .
$$

Далее в описываемом походе сушественно используется быстрое убывание матричных элементов потенциала $V_{n, n^{\prime}}$ с ростом $n$ и $n^{\prime}$, что позволяет "обрезать" матрицу потенциала взаимодействия при некотором числе осцилляторных функций $N$, т.е. считать $V_{n, n^{\prime}}=0$ при $n, n^{\prime}>N$. Таким образом, единственным приближением метода ПГО является замена истинного потеншиала $V$ “усеченным" $\widetilde{V}$, имеюшим конечный ранг:

$$
\widetilde{V}=\sum_{n=0}^{N} \sum_{n^{\prime}=0}^{N} V_{n n^{\prime}}|n l\rangle\left\langle n^{\prime} l\right|
$$


При этом собственная функция полного гамильтониана $H$ ищется в виде

$$
u_{l}(E, r)=\sum_{n=0}^{\infty} a_{n l}(E) R_{n l}(r)
$$

Теперь вместо решения бесконечного набора алгебраических уравнений для коэффициентов $a_{n l}(E)$ (соответствуюших уравнению Шредингера)

$$
\sum_{n^{\prime}=0}^{\infty}\left(H_{n n^{\prime}}^{l}-\delta_{n n^{\prime}} E\right) a_{n^{\prime} l}(E)=0
$$

в случае "усеченного" потенциала (64) можно воспользоваться тем обстоятельством, что при $n, n^{\prime}>N$ матрица гамильтониана $H_{n n^{\prime}}$ переходит в трехдиагональную матрицу кинетической энергии. При этом неизвестные коэффициенты разложения $a_{n l}(E)$ в асимптотической области можно записать в виде [4]-[6]

$$
a_{n l}^{a s}(E)=\cos \delta_{l} S_{n l}(E)+\sin \delta_{l} C_{n l}(E),
$$

где $\delta_{l}(E)$ - точные парциальные фазовые сдвиги, а комплексные коэффициенты $S_{n l}(E)$ и $C_{n l}(E)$ определены в соотношениях (63). Тогда получается конечная система алгебраических уравнений для неизвестных коэффициентов $a_{n l}(E)$ при $n \leqslant N$ :

$$
\sum_{n^{\prime}=0}^{N}\left(H_{n n^{\prime}}^{l}-\delta_{n n^{\prime}} E\right) a_{n^{\prime} l}(E)=-\left(H_{0}^{l}\right)_{N, N+1} a_{N+1, l}^{a s}(E), \quad n=0, \ldots, N
$$

Правые части этих уравнений отвечают сшивке полного и свободного решений на границе потенциала $(n=N)$ в ПГО. Решение системы $(67)$, очевидно, можно записать в виде

$$
a_{n l}(E)=g_{n N} a_{N+1, l}^{a s}(E)
$$

где

$$
g_{n n^{\prime}}=-\sum_{\lambda=0}^{N} \frac{\gamma_{\lambda n}^{*} \gamma_{\lambda n^{\prime}}}{E_{\lambda}-E}\left(H_{0}^{l}\right)_{n^{\prime}, n^{\prime}+1}
$$

$E_{\lambda}$ и $\gamma_{\lambda n}-$ собственные значения и соответствуюшие собственные состояния полной матрицы гамильтониана $\left[H_{0}+\widetilde{V}\right]_{n n^{\prime}}^{l}$ при $n, n^{\prime} \leqslant N$. Далее легко найти наблюдаемые величины, учитьвая тот факт, что коэффициент $a_{N l}(E)$ удовлетворяет одновременно уравнениям (66) и (68).

Таким образом, при использовании ПГО волновые функции рассеяния определяются методом сшивки, но не в координатном пространстве, а в пространстве волновых функций гармонического осциллятора (т.е. по “границе" $n=N$ “усеченного” потенциала).

Некоторой вариацией $J$-матричного подхода является метод, описанный в работе [2], где матрица оператора Грина $G_{l}^{C}(E+i 0)$ гамильтониана $H_{C}$, учитьвающего кулоновское взаимодействие, находится в представлении обобшенных полиномов Лагерра (так 
называемых "кулоновских" функций); при этом снова используется трех диагональный вид матрицы $H_{C}$. Затем “усеченный” потенциал (64) подставляется в уравнение Липпмана-Швингера, что дает неоднородное уравнение для функции рассеяния

$$
\left|\Psi^{(+)}(E, l)\right\rangle=\left|\Phi^{(+)}(E, l)\right\rangle+\sum_{n, n^{\prime}=0}^{N} G_{l}^{c}(E+i 0)|n l\rangle V_{n n^{\prime}}\left\langle n^{\prime} l \mid \Psi^{(+)}(E, l)\right\rangle
$$

или соответствующее однородное уравнение

$$
\left|\Psi_{l}\right\rangle=\sum_{n, n^{\prime}=0}^{N} G_{l}^{c}(E)|n l\rangle V_{n n^{\prime}}\left\langle n^{\prime} l \mid \Psi_{l}\right\rangle
$$

для волновых функций связанных и резонансных состояний. В уравнении (69) $\left|\Phi^{(+)}(E, l)\right\rangle$ - функции, отвечающие рассеянию только на кулоновском потенциале. Домножая уравнения (69) и (70) на “кулоновские" функции $\langle k l|$, получаем матричные уравнения для первых $(N+1)$ коэффициентов разложения точных функций $\left|\Psi^{+}(E, l)\right\rangle$ и $\left|\Psi_{l}\right\rangle$ по "кулоновскому" базису. Таким образом, в этом подходе удается обобщить $J$-матричный метод на решение интегральных уравнений теории рассеяния и сделать его достаточно универсальным для решения задач непрерывного и дискретного спектров.

Существенное отличие этого формализма от предложенного нами метода ПСД заключается в том, что $J$-матричный подход позволяет найти решение задачи рассеяния при произвольной фиксированной энергии. При этом находятся точные (т.е. ненормированные) функции рассеяния. В рамках же подхода ПСД задача рассеяния решается “в ящике", размер и форма которого соответствует ширине $\Delta_{i}$ выбранных энергетических интервалов. При этом вместо полного набора точных функций рассеяния, отвечающих непрерывному спектру энергий, используется конечный набор их нормируемых аналогов $\left|X_{i}\right\rangle$. Более того, вместо точных интегральных операторов теории рассеяния в рамках метода ПС Д строятся их хорошо сходящиеся матричные аналоги, содержашие конечные суммы по дискретизованному континууму, что сильно упрощает все численные процедуры и делает интерпретацию получаемых решений очень легкой.

В целом же можно сказать, что $J$-матричные методы соответствуют нахождению наблюдаемых величин из асимптотических свойств волновых функций, в то время как метод ПС Д отвечает определению наблюдаемых величин из свойств волновых функций во внутренней области. Ясно, что эти формализмы существенно отличаются, хотя и должны приводить к одному и тому же результату в области применимости обоих подходов. $J$-матричный метод, по-видимому, может обеспечивать лучшую сходимость в ряде задач, однако метод ПСД имеет то явное преимушество, что он не привязан к выбору конкретного базиса. Это позволяет, в частности, выбирать наиболее подходяший базис в зависимости от типа решаемых задач. (Подчеркнем, что для многих проблем, таких, например, как рассеяние частицы на притягивающем потенциале с сильным отталкивающим кором или на потенциалах, включающих дальнодействие, выбор осцилляторного базиса в ПГО-подходе заведомо дает плохую сходимость всех разложений). Кроме того, как уже обсуждалось выше, $J$-матричные методы едва ли применимы для неэрмитовых 
операторов взаимодействия. Но самое главное преимушество ПСД-подхода состоит, вероятно, в том, что его многочастичное обобшение довольно легко просматривается, тогда как для $J$-матричных методов оно приводит к существенным и едва ли преодолимым трудностям.

Оба метода можно рассматривать как альтернативные подходы к решению задачи рассеяния в гильбертовом пространстве, но формализм, предложенный в настоящей работе и работе [16], нам представляется более обшим и универсальным.

4.2. Методы построения конечномерных аппроксимаций для оператора Грина и $T$-матрицы. Было бы несправедливо не упомянуть здесь и другие методы, относяшиеся к решению задачи рассеяния с использованием $L_{2}$-базиса, которые также имеют отношение к обсуждаемому здесь подходу. Суть их состоит в построении специальных квадратур для спектрального интеграла, определяюшего функцию Грина, с использованием дискретизации непрерывного спектра гамильтониана $H$ в произвольном базисе. Прежде чем обсуждать взаимосвязь ПСД-подхода и методов построения квадратур для аппроксимации оператора Грина, приведем для удобства читателей краткое описание методов, использующих теорию моментов [10]-[12].

В рамках этих подходов задача обычно формулируется следующим образом. Пусть известно среднее значение точной функции Грина гамильтониана $H$ в некотором локализованном $L_{2}$-состоянии $|\Phi\rangle$ (предположим для простоты, что связанные состояния в системе отсутствуют)

$$
R_{\Phi}(z) \equiv\left\langle\Phi\left|\frac{1}{z-H}\right| \Phi\right\rangle=\int_{0}^{\infty} d E^{\prime} \frac{\left|\left\langle\Phi \mid \Psi\left(E^{\prime}\right)\right\rangle\right|^{2}}{z-E^{\prime}},
$$

а также среднее значение

$$
\bar{R}_{\Phi}(z) \equiv\left\langle\Phi\left|\frac{1}{z-\bar{H}}\right| \Phi\right\rangle=\sum_{i=1}^{N} \frac{\left|\left\langle\Phi \mid Y_{i}\right\rangle\right|^{2}}{z-E_{i}^{*}},
$$

отвечающее конечной матрице гамильтониана $\bar{H}=[H]_{n n^{\prime}}$ в произвольном дискретном базисе $\left(n, n^{\prime} \leqslant N\right)$. Здесь $\left|Y_{i}\right\rangle$ и $E_{i}^{*}$ - собственные функции и собственные значения матрицы $\bar{H}$. Обозначим

$$
\rho_{\Phi}(E) \equiv|\langle\Phi \mid \Psi(E)\rangle|^{2}, \quad \rho_{i} \equiv\left|\left\langle\Phi \mid Y_{i}\right\rangle\right|^{2},
$$

где $\rho_{\Phi}(E)$ представляет собой непрерьвную по энергии функцию плотности состояний, а $\rho_{i}-$ ее дискретный аналог.

Если переменная $z$ комплексная и $\operatorname{Im} z \neq 0$, сумму (72) можно рассматривать как квадратурную аппроксимацию интеграла (71), т.е. считать

$$
R_{\Phi}(z) \approx \bar{R}_{\Phi}(z), \quad \operatorname{Im} z \neq 0 .
$$

Сложности появляются в случае $\operatorname{Im} z \rightarrow 0$, поскольку функция $R_{\Phi}(z)$ имеет кинематический разрез вдоль действительной оси, скачок на котором определяется из предельной формулы

$$
R_{\Phi}(E \pm i 0)=\mathcal{P} \int_{0}^{\infty} d E^{\prime} \frac{\rho_{\Phi}\left(E^{\prime}\right)}{E-E^{\prime}} \mp i \pi \rho_{\Phi}(E),
$$


а функция $\widehat{R}_{\Phi}(z)$ вместо разреза содержит только полюсы $z=E_{i}^{*}$. Основная проблема заключается в нахождении мнимой части $R_{\Phi}(z)$, т.е. функции плотности состояний. Достаточно гладкую аппроксимацию функции $\rho_{\Phi}(E)$ можно построить, используя моменты распределения энергии, отвечаюшие этой функции плотности. Для этих целей можно использовать дискретизованный спектр гамильтониана $H$

$$
\mu_{k}=\int_{0}^{\infty} E^{k} \rho_{\Phi}(E) d E \approx \sum_{i=1}^{N} \rho_{i} E_{i}^{* k}, \quad k=0, \ldots, 2 M-1 .
$$

Зная моменты $\mu_{k}$ распределения энергии, можно на основе метода моментов построить квадратуру для регулярного интеграла вида

$$
\int d E \rho_{\Phi}(E) F(E) \approx \sum_{n=1}^{M} \rho_{n}^{(M)} F\left(\epsilon_{n}^{(M)}\right),
$$

если аппроксимировать, как обычно, подынтегральную функцию $F(E)$ посредством полинома степени $2 M$. Узлы $\epsilon_{n}^{(M)}$ и веса $\rho_{n}^{(M)}$ такой квадратуры определяются из системы нелинейных уравнений

$$
\mu_{k}=\sum_{n=1}^{M}\left(\epsilon_{n}^{(M)}\right)^{k} \rho_{n}^{(M)}, \quad k=0, \ldots, 2 M-1,
$$

решение которых подробно описано в работах [10], [12]. Заметим, что поскольку интеграл, определяюший реальную часть $R_{\Phi}$, не является регулярным, обычная теория моментов к нему неприменима и нужно строить специальные квадратуры.

В работе [10] строится гистограммная аппроксимация Стилтьеса для функции $f(E)$, определяемой соотношением $d f(E)=\rho_{\Phi}(E) d E$, следуюшего вида:

$$
\begin{array}{llrl}
f_{s}^{(M)}(E) & =0, & & 0 \leqslant E<\epsilon_{1}^{(M)}, \\
f_{s}^{(M)}(E)=\sum_{n=1}^{j} \rho_{n}^{(M)}, & \epsilon_{j}^{(M)}<E<\epsilon_{j+1}^{(M)}, \\
f_{s}^{(M)}(E)=\sum_{n=1}^{M} \rho_{n}^{(M)}=\mu_{0}, & \epsilon_{M}^{(M)}<E .
\end{array}
$$

Эта аппроксимация удовлетворяет неравенствам Чебышева и сходится к точной функции $f(E)$. Соответствуюшее приближение для самой функции плотности $\rho_{\Phi}(E)$ получается путем дискретного дифференцирования соотношений (77):

$$
\begin{array}{ll}
\rho_{\Phi}^{(M)}(E)=\frac{1}{2} \frac{\rho_{1}^{(M)}}{\epsilon_{1}^{(M)}}, & 0 \leqslant E<\epsilon_{1}^{(M)}, \\
\rho_{\Phi}^{(M)}(E)=\frac{1}{2} \frac{\rho_{n}^{(M)}+\rho_{n+1}^{(M)}}{\epsilon_{n+1}^{(M)}-\epsilon_{n}^{(M)}}, & \epsilon_{n}^{(M)}<E<\epsilon_{n+1}^{(M)}, \\
\rho_{\Phi}^{(M)}(E)=0, & \epsilon_{n}^{(M)}<E,
\end{array}
$$

6 Теоретическая и математическая физика, т. 134, № 3, 2003 г. 
причем величина $\rho_{\Phi}^{(M)}(E)$ сходится к исходной функции плотности, если последняя непрерывна.

Для реальной части функции $R_{\Phi}(E+i 0)$ строится гистограммная аппроксимация [10]

$$
\operatorname{Re} R_{\Phi}\left(\epsilon_{i}^{*(M)}+i 0\right)=\mathcal{P} \int_{0}^{\infty} d E^{\prime} \frac{\rho_{\Phi}^{(M)}(E)}{\epsilon_{i}^{*(M)}-E}=\sum_{n=1}^{M-1} \rho_{\Phi}^{(M)}\left(\epsilon_{i}^{*(M)}\right) \ln \left|\frac{\epsilon_{i}^{*(M)}-\epsilon_{n}^{(M)}}{\epsilon_{i}^{*(M)}-\epsilon_{n+1}^{(M)}}\right|
$$

где $\epsilon_{i}^{*(M)}=\left(\epsilon_{i}^{(M)}+\epsilon_{i-1}^{(M)}\right) / 2$.

В работе [10] строится также аппроксимация Чебышева для $\rho_{\Phi}(E)$, непрерывная по $E$, для чего решается проблема моментов (76) при каждом значении $E$ с дополнительным условием на один из узлов квадратуры $\epsilon_{0}(E)=E$. Аппроксимация Чебышева является $(2 M-4)$ раз дифференцируемой функцией энергии $E$.

Заметим, что формула (79) очевидным образом совпадает с формулой (15) для среднего значения оператора Грина в ПСД $\operatorname{Re}\left\langle\Phi\left|G_{0 P}^{( \pm)}\left(\mathcal{E}_{i}^{*}\right)\right| \Phi\right\rangle$, если узловые точки квадратуры и разбиения (3) совпадают, т.е. если $\epsilon_{n}^{(M)}=\mathcal{E}_{n}$ и

$$
\rho_{\Phi}^{(M)}\left(\mathcal{E}_{n}^{*}\right)=\frac{\left|\left\langle X_{n} \mid \Phi\right\rangle\right|^{2}}{\Delta_{n}}
$$

Как видим, последнее соотношение почти соответствует (78). Таким образом, два совершенно разных подхода к нахождению конечномерных аппроксимаций для функций Грина приводят к обшему результату. На самом деле это обстоятельство следует из того, что для $L_{2}$-функции $|\Phi\rangle$ будет приблизительно верно соотношение

$$
\left\langle\Phi\left|\widehat{G}_{0}^{( \pm)}(E)\right| \Phi\right\rangle \approx\left\langle\Phi\left|P \widehat{G}_{0}^{( \pm)}(E) P\right| \Phi\right\rangle
$$

при достаточно малых ширинах энергетических интервалов.

Возможны и другие вариации применения метода моментов для построения непрерывной функции плотности (см. [11]), но обшим для всех этих подходов является использование дискретизованного спектра гамильтониана $H$ лиш для определения моментов распределения энергии. Упомянем в этой связи также нашу работу [15], в которой независимо была построена аппроксимация для функции плотности $\rho_{\Phi}(E)$ по формулам, практически совпадаюшим с (78), если в качестве узлов и весов квадратуры использовать параметры исходного спектра дискретизованной задачи $\left\{E_{i}^{*}, \rho_{i}\right\}$.

$\mathrm{K}$ настояшему времени развито также множество иных методов нахождения дискретизованных представлений для функции Грина и $T$-матришы. К ним относится, например, алгоритм Швингера-Ланцоша [13], основанный на вариационном принципе Швингера, согласно которому элемент внемассовой $T$-матрицы

$$
T_{f i} \equiv\left\langle\Psi_{0}\left(E_{f}\right)|T(E)| \Psi_{0}\left(E_{i}\right)\right\rangle=\left\langle\Psi_{0}\left(E_{f}\right)\left|V\left(V+V G_{0} V\right)^{-1} V\right| \Psi_{0}\left(E_{i}\right)\right\rangle
$$

является стационарным значением функционала $T\left[\Psi^{(-)}, \Psi^{(+)}\right]$, достигаемым в случае, когда в качестве $\left|\Psi^{( \pm)}\right\rangle$берутся решения соответствуюшего уравнения ЛиппманаШвингера, отвечаюшие граничным условиям в виде сходяшихся и расходяшихся волн. 
Если разложить каждую из этих функций по некоторым конечным базисам $\left\{\left|g_{k}^{( \pm)}\right\rangle, k=\right.$ $1, N\}$ (которые нужно найти)

$$
\left|\Psi^{( \pm)}\right\rangle=\sum_{k=1}^{N} C_{k}^{( \pm)}\left|g_{k}^{( \pm)}\right\rangle
$$

то получится значение функционала, стационарное относительно вариации коэффициентов $C_{k}^{( \pm)}$:

$$
T_{f i}^{N}=\sum_{k, l}^{N}\left\langle\Psi_{0}\left(E_{f}\right)|V| g_{k}^{(+)}\right\rangle\left(M^{-1}\right)_{k l}\left\langle g_{l}^{(-)}|V| \Psi_{0}\left(E_{i}\right)\right\rangle
$$

где $\mathbf{M}^{-1}$ - матрица, обратная $M_{k l}=\left\langle g_{k}^{(-)}\left|V+V G_{0} V\right| g_{k}^{(+)}\right\rangle$. Суть метода состоит в том, что, используя алгоритм Ланцоша, можно построить искомые базисные векторы $\left|g_{k}^{( \pm)}\right\rangle$ (на основе простых рекуррентных соотношений) таким образом, что матрища М будет трехдиагональной. В этом случае для нахождения элементов $T_{f i}^{N}$ потребуется только один элемент $\left(M^{-1}\right)_{11}$, определяемый с помощью непрерьвной дроби. Таким путем авторам [13] удалось развить очень экономичный и простой алгоритм нахождения аппроксимаций для элементов внемассовой $T$-матрицы, позволяющий делать весьма точные расчеты в случае нелокальных и зависяших от энергии операторов взаимодействия. Кроме того, там же было показано, что полученное выражение для $T_{f i}^{N}$ формально эквивалентно конечномерной аппроксимации, получаемой с помощью преобразования Шенкса [20], действующего на конечные суммы ряда теории возмушений для внемассовой $T$-матрицы. Это преобразование позволяет ускорить сходимость частичных сумм ряда. Численно оно осуществляется при использовании эпсилон-алгоритма Винна [21]. Интересным является то обстоятельство, что хотя можно показать эквивалентность аппроксимаций, полученных по двум описанным алгоритмам, при численных расчетах в некоторых случаях их сходимости будут сильно различаться.

Итак, методы, описанные в этом разделе, позволяют найти хорошо сходящиеся численные аппроксимации для функции Грина или $T$-матрицы в произвольном базисе (упомянем также $R$-матричный подход и его многочисленные вариации (см. [14])). Однако нам не известны какие-либо обобщения этих частных подходов на многомерный или многочастичный случаи. Отличие выражения (15), найденного в рамках обсуждаемого здесь формализма ПСД, от строящихся разными методами конечномерных аналогов оператора Грина состоит в том, что для его построения в нашем подходе используется явная связь между точными функциями рассеяния и их СД. Заметим, что полученное нами представление для оператора Грина можно считать также некоторой квадратурой, однако ее узлы и веса непосредственно привязаны к локальным свойствам непрерывного спектра (т.е. к С Д функций рассеяния), а не к моментам распределения энергии по всему непрерывному спектру, как в подходе, описанном в работе [11]. Однако главное, что выделяет метод ПСД среди других методов дискретизации континуума, - это то обстоятельство, что аппроксимация функции Грина в нашем подходе является лишш одним из многих элементов внутренне-согласованного формализма, представляющего по сути "дискретизованную" теорию рассеяния. 


\section{5. ЗАКЛЮЧЕНИЕ}

В данной работе мы завершили (на уровне теории двухчастичного рассеяния) развитие общего подхода к дискретизации континуума на основе техники ПСД. Этот метод, являясь оригинальным по сушеству, тем не менее имеет, как показано выше, ряд обших черт с другими методами решения квантовых задач в континууме в $L_{2}$-базисе, предложенными к настояшему моменту. Некоторые детали этих взаимосвязей были обсуждены в разделе 4 данной работы. Сильной стороной всех этих методов является принципиальная простота учета (одномерного) континуума, сводящаяся в основном к однократной диагонализации матрицы гамильтониана в каком-либо подходящем базисе. Это позволяет рассматривать сложные нелокальные взаимодействия почти так же просто, как и локальные потенциалы. Но метод ПС Д позволяет продвинуться на этом "матричном" пути еше дальше и включить также комплексные и зависящие от энергии взаимодействия, что фактически делает такой подход универсальной схемой дискретизации континуума в квантовой теории рассеяния.

Дополнительным аргументом в пользу этого утверждения является тот факт, что при использовании волновых пакетов, построенных из функций свободного движения, просматривается также и многочастичное обобшение этого метода. Здесь уместно привести аргументы, подкрепляюшие это важное утверждение.

Во-первых, хорошо известно, что ставшая классической на сегодняшний день фаддевская редукция (позволяюшая свести многочастичные интегральные уравнения Липпмана-Швингера, ядра которых не удовлетворяют условию Фредгольма, к “фредгольмовским" уравнениям Фаддеева-Якубовского) приводит к несингулярным в принципе и ограниченным интегральным ядрам, т.е. локализованным в многомерном конфигурационном пространстве по всем радиальным координатам. Иными словами, важнейшее свойство связности всех диаграмм, представляюших ряд многократных перерассеяний для уравнений Фаддеева, как очевидно, непосредственно ведет к "локализации" соответствуюших этому ряду операторов в конфигурационном пространстве (под термином "локализация" мы понимаем здесь затухание интегральных ядер вдоль всех независимых радиальных координат). Проще всего это свойство локализации можно продемонстрировать с помошью обшего определения оператора перехода $\mathcal{T}_{a b}$ (см. [22]) через полную резольвенту системы $G$ :

$$
\mathcal{T}_{a b}=\bar{V}_{a}+\bar{V}_{a} G \bar{V}_{b},
$$

где $a$ и $b$ - два разбиения системы из $N$ частиц, отвечаюшие начальному $(a)$ и конечному (b) каналам реакции. Операторы $\bar{V}_{a}$ и $\bar{V}_{b}$, как обычно, представляют внешнее взаимодействие в соответствуюших каналах (т.е. $H=H_{0}+V_{a}+\bar{V}_{a}=H_{0}+V_{b}+\bar{V}_{b}$, где $V_{a}$, $V_{b}$ - "внутренние" взаимодействия в каналах $(a)$ и $\left.(b)\right)$.

Если теперь выполнить фаддевскую редукцию для полной резольвенты системы $G$ [22] и написать итерационный ряд для получившейся системы интегральных уравнений для компонент $G^{(a)}$ полной резольвенты, а затем подставить этот ряд в общее определение (82), то легко видеть, что все диаграммы ряда перерассеяний, представляющие оператор перехода $\mathcal{T}_{a b}$, не только будут связными (кроме свободной резольвенты $G_{0}$ ), но 
и дадут затухаюшее поведение по радиальным переменным во входном и выходном каналах. Таким образом, аппроксимация точных многочастичных функций рассеяния, удовлетворяюших интегральным уравнениям Фаддеева-Якубовского, посредством их СД, локализованных в пространстве, должна быть столь же точной, как и в двухчастичном случае (т.е. как для произведения $G_{0} V \Psi$ в случае двухчастичного уравнения Липпмана-Швингера).

Во-вторых, поскольку в ПСД-подходе рецепт перехода от плоских волн к свободным волновым пакетам (локализованным в пространстве) кажется универсальным, то можно прямо применить его к интегральным уравнениям Фаддеева-Якубовского и найти их конечномерные матричные аналоги. Одно известное обстоятельство делает этот подход в высокой степени реализуемым. Оно состоит в том, что при использовании, скажем, сепарабельной формы парного потенциала уравнения Фаддеева сводятся, как хорошо известно [22], к одномерному уравнению типа Липпмана-Швингера с нелокальным и зависящим от энергии потенциалом взаимодействия. А этот случай как раз и вписьвается в описанную в данной работе схему ПСД (в отличие от $J$-матричного подхода или методов квадратурной аппроксимации).

ПРИЛОЖЕНИЕ

В случае комплексного потенциала взаимодействия полный гамильтониан не является эрмитовым, поэтому уравнения для in- и out-состояний отличны от (17) (см. [23]):

$$
\begin{aligned}
& \left|\Psi^{(+)}(E, l)\right\rangle=\left|\Psi_{0}(E, l)\right\rangle+G_{0}^{(+)}(E) V\left|\Psi^{(+)}(E, l)\right\rangle, \\
& \left|\Psi^{(-)}(E, l)\right\rangle=\left|\Psi_{0}(E, l)\right\rangle+G_{0}^{(-)}(E) V^{\dagger}\left|\Psi^{(-)}(E, l)\right\rangle .
\end{aligned}
$$

Тогда элементы $S$-матрищы будут определяться так же, как и в случае вешественного потенциала:

$$
\left\langle\Psi^{(-)}(E, l) \mid \Psi^{(+)}\left(E^{\prime}, l\right)\right\rangle=S_{l}(E) \delta\left(E-E^{\prime}\right) .
$$

Наряду с функциями $\left|\Psi^{(-)}\right\rangle$и $\left|\Psi^{(+)}\right\rangle$можно определить биортогональные наборы состояний

$$
\begin{aligned}
& \left|\tilde{\Psi}^{(+)}(E, l)\right\rangle=\left|\Psi_{0}(E, l)\right\rangle+G_{0}^{(+)}(E) V^{\dagger}\left|\tilde{\Psi}^{(+)}(E, l)\right\rangle, \\
& \left|\tilde{\Psi}^{(-)}(E, l)\right\rangle=\left|\Psi_{0}(E, l)\right\rangle+G_{0}^{(-)}(E) V\left|\tilde{\Psi}^{(-)}(E, l)\right\rangle .
\end{aligned}
$$

Таким образом, будут верны соотношения ортогональности

$$
\left\langle\tilde{\Psi}^{(+)}(E, l) \mid \Psi^{(+)}\left(E^{\prime}, l\right)\right\rangle=\delta\left(E-E^{\prime}\right)
$$

или

$$
\left\langle\tilde{\Psi}^{(-)}(E, l) \mid \Psi^{(-)}\left(E^{\prime}, l\right)\right\rangle=\delta\left(E-E^{\prime}\right) .
$$

Можно показать [23], что все четыре набора функций в случае одноканального рассеяния связаны с функциями $|\Psi(E, l)\rangle$, отвечающими граничным условиям в форме стоячих волн, соотношениями

$$
\begin{array}{ll}
\left|\Psi^{(+)}(E, l)\right\rangle=e^{i \delta_{l}}|\Psi(E, l)\rangle, & \left|\tilde{\Psi}^{(+)}(E, l)\right\rangle=e^{i \delta_{l}^{*}}|\Psi(E, l)\rangle ; \\
\left|\Psi^{(-)}(E, l)\right\rangle=e^{-i \delta_{l}^{*}}|\Psi(E, l)\rangle, & \left|\tilde{\Psi}^{(-)}(E, l)\right\rangle=e^{-i \delta_{l}}|\Psi(E, l)\rangle,
\end{array}
$$


где $\delta_{l}$ - фазовые сдвиги. (Сами функции $|\Psi(E, l)\rangle$ не являются вешественными, но отвечают вещественному соотношению ортогональности $\left\langle\Psi(E, l) \mid \Psi\left(E^{\prime}, l\right)\right\rangle=\delta\left(E-E^{\prime}\right)$.

В рамках формализма ПС Д можно определить наборы С Д функций рассеяния $\left|Y_{i}^{( \pm)}\right\rangle$ и $\left|\widetilde{Y}_{i}^{( \pm)}\right\rangle$, а также четыре набора $P$-проекций $\left|\mathcal{Y}^{( \pm)}(E)\right\rangle$. Используя соотношения (П.3), можно показать, что для $S$-оператора в ПС Д будет верно разложение (51), где элементы $S_{i}$ определяются следуюшим образом:

$$
S_{i} \approx \frac{\left\langle\widetilde{\mathcal{Y}}^{(-)}\left(\mathcal{E}_{i}^{*}\right) \mid \mathcal{Y}^{(+)}\left(\mathcal{E}_{i}^{*}\right)\right\rangle}{\left\langle\widetilde{\mathcal{Y}}^{(-)}\left(\mathcal{E}_{i}^{*}\right) \mid \widetilde{\mathcal{Y}}^{(-)}\left(\mathcal{E}_{i}^{*}\right)\right\rangle} .
$$

Легко видеть, что функции $\left|\widetilde{\mathcal{Y}}_{i}^{(-)}\left(\mathcal{E}_{i}^{*}\right)\right\rangle$ выражаются через элементы матриц $\boldsymbol{\Omega}^{(-)}\left(\mathcal{E}_{i}^{*}\right)$, определяемых точно так же, как и в случае вешественного потенциала, т.е. для $S_{i}$ будет верно равенство $(29)$, где матрицы $\boldsymbol{\Omega}^{( \pm)}\left(\mathcal{E}_{i}^{*}\right)$ определяютя согласно (22) (совершенно аналогично вещественному случаю).

Благодарности. Авторы благодарят проф. Р. Джонсона, проф. И. Томпсона и проф. Дж. Тостевина, а также других сотрудников теоретической группы университета Surrey (Великобритания) за полезные обсуждения. Данная работа выполнена при частичной финансовой поддержке РФФИ, грант №01-02-16621.

\section{Список литературы}

[1] E. J. Heller. Phys. Rev. A. 1975. V. 12. P. 1222.

[2] B. Konya, G. Levai, Z. Papp. Phys. Rev. C. 2000. V. 61. P. 034302.

[3] Г. Ф. Филиппов, И. П. Охрименко. ЯФ. 1980. Т. 32. С. 932.

[4] Ю. И. Нечаев, Ю. Ф. Смирнов. ЯФ. 1982. Т. 35. С. 1385.

[5] J. M. Bang, A. I. Mazur, A. M. Shirokov. Yu. F. Smirnov, S. A. Zaytsev. Ann. Phys. 2000. V. 280. P. 299.

[6] А. М. Широков, Ю. Ф. Смирнов, С. А. Зайщев. ТМФ. 1998. Т. 117. С. 227.

[7] H. A. Yamani, M. S. Abdelmonem. J. Phys. B. 1997. V. 30. P. 1633.

[8] T. Vertse, P. Curutchet, R. J. Liotta. Phys. Rev. C. 1990. V. 42. P. 2605; T. Vertse, P. Curutchet, R. J. Liotta, J. Bang, N. Van Giai. Phys. Lett. B. 1991. V. 264. P. 1 R. J. Liotta, E. Maglione, N. Sandulescu, T. Vertse. Phys. Lett. B. 1996. V. 367. P. 1.

[9] E. J. Heller, W. P. Reinhardt, H. A. Yamani. J. Comput. Phys. 1973. V. 13. P. 536.

[10] C. T. Corcoran, P. W. Langhoff. J. Math. Phys. 1977. V. 18. P. 651.

[11] J. R. Winick, W. P. Reinhardt. Phys. Rev. A. 1978. V. 18. P. 910.

[12] I. Cacelli, V. Carravetta, A. Rizzo. J. Chem. Phys. 1993. V. 98. P. 8742.

[13] M. Cizek, J. Horacek, H.-D. Meyer. Comput. Phys. Commun. 2000. V. 131. P. 41.

[14] G. Melnikov, J. Horacek, H. Nakamura. Comput. Phys. Commun. (in press).

[15] О. А. Рубцова, В. И. Кукулин. ЯФ. 2001. Т. 64. С. 1882.

[16] О. А. Рубцова, В. И. Кукулин. ТМФ. 2002. Т. 130. С. 64.

[17] А. Мессиа. Квантовая механика. М.: Наука, 1978.

[18] Р. Ньютон. Теория рассеяния волн и частиц. М.: Мир, 1969.

[19] С. Сунакава. Квантовая теория рассеяния. М.: Мир, 1979.

[20] D. Shanks. J. Math. Phys. 1955. V. 34. P. 1.

[21] P. Wynn. Math. Tables Aids Comput. 1956. V. 10. P. 91.

[22] С. П. Меркурьев, Л. Д. Фаддеев. Квантовая теория рассеяния для систем нескольких частиц. М.: Наука, 1985.

[23] H. Feshbach. Ann. Phys. 1985. V. 164. P. 398. 